DOI: $10.24850 /$ j-tyca-2020-05-05

Artículos

\title{
Un nuevo criterio para la estimación de rugosidad compuesta en modelos hidráulicos
}

\section{A new criteria for estimating composite roughness on hydraulic models}

Humberto Marengo ${ }^{1}$

Álvaro A. Aldama²

Ignacio Romero 3

${ }^{1}$ Comisión Internacional de Límites y Aguas México-Estados Unidos, Ciudad Juárez, Chih., México, hmarengom@gmail.com

${ }^{2}$ Consultor independiente, Cuernavaca, México, alvaro.aldama@gmail.com

${ }^{3}$ Comisión Federal de Electricidad, Ciudad de México, México, ignacio.romero02@cfe.gob.mx

Autor para correspondencia: Humberto Marengo, hmarengom@gmail.com

\section{Resumen}


La ingeniería práctica ha considerado a la ecuación de Colebrook-White (1939) como la más apropiada para estimar el coeficiente de fricción en un régimen turbulento de transición y los que presentan turbulencia completamente desarrollada. Debido a la dificultad que significa tener dicho coeficiente implícito en ella, se ha solucionado este hecho por parte de la profesión con diversos métodos numéricos para flujos que se presentan en conductos fabricados, en su mayoría con un solo material. En conducciones con más de dos materiales (donde se presentan rugosidades compuestas), algunos autores han desarrollado criterios que emplean otro tipo de soluciones, como la presentada por Elfman (2005), que se basa en el criterio de Nikuradse (1933). En este artículo se plantea un nuevo criterio que permite el cálculo de la rugosidad compuesta en túneles con sección baúl utilizando la ecuación de Colebrook. Se parte de la experimentación realizada por los autores, que se hizo para la construcción de diversas obras de desvío en presas mexicanas (El Cajón y La Yesca); se muestra la aplicación en los modelos hidráulicos que se construyeron; se concluyó que es una metodología más científica y aplicable para el diseño de este tipo de obras.

Palabras clave: rugosidad compuesta, modelos hidráulicos, ecuación de Colebrook.

\section{Abstract}

Practical engineering has considered that implicit Colebrook-White equation (1939) has been the standard for estimating pipe friction factor in a fully developed turbulent regime in conductions manufactured with 
one material. Due to the implicit character of the criteria, several authors have developed some criteria that use other kind of solutions like presented by Elfman (2005) who takes as basis Nikuradse criteria. In this study, a new methodology is proposed with basis in Colebrook equation for tunnels with composite roughness. Considering hydraulic models build by the authors for diversion works (El Cajón and La Yesca), the analysis is made, and is concluded that this Colebrook analysis is more scientific, and applicable to this kind of works.

Keywords: Compound roughness, Hydraulic Models, Colebrook equation.

Recibido: 23/05/2019

Aceptado: $18 / 02 / 2020$

\section{Introducción}

Esta investigación tiene como origen el análisis de riesgo de falla por desbordamiento del proyecto hidroeléctrico Aguamilpa realizado por Marengo (2006), donde se plantea que si los túneles de desvío se 
hubieran construido con rugosidades compuestas, se habría alcanzado hasta un $50 \%$ más en la confiabilidad del funcionamiento hidráulico de la obra en conjunto. El concepto de rugosidades compuestas en este caso se define como el hecho de colocar una plantilla de concreto hidráulico en el piso y concreto lanzado en paredes y bóveda de los túneles de sección tipo baúl.

Al hacer una revisión del análisis de conducciones con base en rugosidades compuestas, se encontró que se mencionan en Chow (1959), USBR (1992), y en Yen (2002), quien hace un planteamiento teórico de la capa límite y la superficie de resistencia de las paredes del flujo; compara la energía de los coeficientes de resistencia con respecto al momentum; en cuanto a las rugosidades compuestas, menciona que hay 17 ecuaciones para su evaluación, en las que no está definido cuáles de éstas - con sus respectivas subsecciones en las que se dividen- son las más adecuadas ni qué fórmulas nuevas se pueden plantear con base en la mecánica de fluidos, y establece que debe ser investigado.

Considerando lo anterior, es necesario tomar en cuenta que la rugosidad en túneles excavados en roca sin revestimiento está gobernada por la estructura de la roca y la técnica con que se excavó el túnel (voladuras convencionales hacia aguas arriba o hacia aguas abajo, y hoy en día, con máquinas tuneleras, también Ilamadas TBM, por sus siglas en inglés). En algunas ocasiones, la estructura de la roca hace que la distribución de la rugosidad sea extremadamente irregular; sin embargo, si el macizo rocoso es denso y de buena calidad sin un fracturamiento frecuente de la roca, la rugosidad de los túneles puede ser uniforme a lo largo de su trayectoria, lo cual sucede en pocas ocasiones. De cualquier 
modo, los túneles siempre presentarán irregularidades y protuberancias, con lo que la rugosidad de los mismos usualmente se considerará por lo menos como una "superficie ligeramente ondulada".

Por esta razón es común que el cálculo de las pérdidas de carga en prototipos se haga por medio de las ecuaciones de Manning, Chezy y Colebrook; pero en opinión de Yen, no hay claridad ni ventajas teóricas de un coeficiente sobre otro, por lo que una comparación de los tres coeficientes en un problema específico puede ser muy útil.

Históricamente, la estimación del coeficiente de fricción $\lambda$ tiene la ventaja de estar relacionada con el desarrollo de la mecánica de fluidos por parte de los científicos y en ocasiones es minimizado para aplicaciones prácticas por parte de los ingenieros, ya que la ecuación de Chezy —que es más simple-que la de Manning tiene la ventaja de ser constante en todos los casos de flujo turbulento.

Desde el punto de vista del diseño de túneles hidráulicos, en Noruega se han desarrollado estudios (Czarnota, 1986; Elfman, 2005), que caracterizan el flujo de agua con base en las ecuaciones de Nikuradse (1933), pretendiendo definir el comportamiento hidráulico de los túneles al considerar sólo la rugosidad de los mismos $\left(K_{S}\right)$; sin embargo, esto no es del todo válido, pues además de la propia rugosidad relativa para estimar las pérdidas de carga, también interviene el factor de fricción que representa las características del escurrimiento.

La principal dificultad de emplear la ecuación de Colebrook-White es que conlleva la estimación del factor de fricción de manera implícita, y entonces requiere de un proceso iterativo para su estimación. La solución de un problema necesita de una gran cantidad de cálculos y simulaciones 
que no siempre resultan aplicables de forma sencilla. De hecho, el uso de la gráfica de Moody como alternativa elimina el requerimiento de las iteraciones; con todo, es una herramienta gráfica que no resulta conveniente para cálculos computacionales.

Para conocer el comportamiento hidráulico de los túneles con estas condiciones de rugosidad, tratando de encontrar la validación que se requiere, se construyeron modelos hidráulicos (Marengo, 2016) en los que para su estimación se partió del criterio propuesto por Elfman (2005), que como se señaló, toma como base las ecuaciones de Nikuradse, quien parte de adoptar como premisa que la velocidad máxima en las secciones estudiadas con sección baúl se presenta en el centro de las secciones analizadas, lo cual es aplicable sólo a tubos circulares, lo que se considera que no es del todo exacto si las secciones analizadas no poseen esta geometría.

Al aplicar la ecuación de Colebrook-White se ha identificado como aquello con más validez para conocer de una manera más asertiva el comportamiento del flujo en un régimen de transición y turbulento; en este artículo se desarrolla tal criterio, el cual permite estimar el comportamiento hidráulico de los túneles al operar como tubo lleno en modelos (que es poco frecuente en la literatura técnica), y se pretende validarlo en los modelos hidráulicos estudiados, dejando para investigaciones futuras su comprobación en prototipos.

Con dicha perspectiva, este trabajo se presenta como sigue: primero se plantean las ecuaciones básicas que rigen el fenómeno, se comenta entonces el modelado hidráulico que se hizo considerando cuatro materiales diferentes y se introduce el nuevo criterio de análisis; se 
comparan los resultados obtenidos para el caso acrílico-lija, acrílicoplástico y acrílico-carpeta, y por último se muestra un análisis de sensibilidad basado en el criterio de Bombardelli (2003), con lo que se emiten las conclusiones correspondientes.

\section{Revisión de las ecuaciones de resistencia y coeficientes de rugosidad}

Al analizar el escurrimiento de fluidos en diferentes conducciones es conveniente aclarar la diferencia entre coeficiente de resistencia y coeficiente de rugosidad, los que, por lo general, de manera equivocada se consideran con la misma óptica. El coeficiente de rugosidad es una medida geométrica que refleja los desniveles de la frontera geométrica. Un coeficiente de resistencia es una medida que refleja el comportamiento dinámico del flujo en términos del momentum o la energía en la frontera de la conducción que transporta el fluido. En la mecánica de fluidos, el coeficiente de resistencia se considera como un fenómeno debido al momentum del flujo; en la ingeniería civil es usual que los coeficientes de resistencia sean tratados desde el punto de vista de la energía del flujo. Un análisis cuidadoso y detallado de ambos conceptos (Yen, 2002) establece que sólo pueden compararse para el caso de flujo uniforme y 
permanente en canales o conductos prismáticos con paredes rígidas, como es el caso de los túneles que aquí se estudian.

La fuerza tractiva $\tau$, según Leopardi (2005) producida por un flujo entre dos secciones es proporcional al gradiente de energía $I=\Delta h / L$ y es expresada como:

$\tau=\gamma R I$

Para el flujo turbulento se establece que $\tau$ depende de la densidad $\rho=\gamma / g$, de la velocidad media $V$, el radio hidráulico $R$ y la rugosidad absoluta $K$ :

$\tau=f(\rho, V, R, K)$

Que es el teorema de Buckingham y se puede expresar como:

$\tau=\frac{\lambda}{8} V^{2}$

Sustituyendo la Ecuación (3) en la Ecuación (1), el gradiente de energía se expresa como: 
Tecnología y

Ciencias $\stackrel{\unlhd}{\unlhd}$ gua
2020, Instituto Mexicano de Tecnología del Agua

Open Access bajo la licencia CC BY-NC-SA 4.0

(https://creativecommons.org/licenses/by-nc-sa/4.0/)

$I=\frac{\lambda V^{2}}{g R}$

La pérdida por fricción entre dos secciones puede expresarse y resolverse numéricamente con la ecuación del momentum $(d h / d s)+\Delta=0$, donde $h$ es el trinomio de Bernoulli; en consecuencia, entre dos secciones:

$Z_{1}+h_{1}+\alpha_{1} V_{1}^{2} / 2 g=Z_{2}+h_{2}+\alpha_{2} V_{2}^{2} / 2 g+\Delta$

Donde los índices 1 y 2 especifican la sección considerada. De la Ecuación (3):

$$
\lambda=\frac{8 \tau}{\rho V^{2}}=\frac{8 g R I}{\gamma V^{2}}
$$

Con estas expresiones fue posible calcular los parámetros $I, \tau, \gamma$ para los diferentes gastos estudiados en cada modelo.

Cuando se operan modelos hidráulicos, la mayoría cae en la zona de transición del Ábaco de Moody, por lo que los números de Reynolds estudiados son $R_{e}>25000$. Por ello se utilizó el criterio de rugosidad de Colebrook (Yen, 2002), que se expresa como:

$$
\frac{1}{\sqrt{\lambda}}=-2 \log \left(\frac{2 K c}{K_{2} R}+\frac{K_{3}}{4 R e \sqrt{\lambda}}\right)
$$


Para un tubo circular, las constantes de la ecuación de Colebrook $\left(\right.$ Yen, 2002) son $K_{1}=2.00, K_{2}=14.83, K_{3}=2.52$, donde:

$R_{e}=\frac{V R}{v}$

$V=$ velocidad media de la sección transversal

$R=$ radio hidráulico

$v=$ viscosidad cinemática del flujo.

En una sección baúl típica se considera que el radio hidráulico, (Figura $1, R=b / 2$ ):

$R=\frac{A}{P}=\frac{b^{2}(4+\pi)}{b(4+\pi)}$

$\Rightarrow 4 R_{e}=4 \frac{V b}{2 v}=2 \frac{V b}{v}$ 
Tecnología y

Ciencias $₫$ Agua
2020, Instituto Mexicano de Tecnología del Agua

Open Access bajo la licencia CC BY-NC-SA 4.0

(https://creativecommons.org/licenses/by-nc-sa/4.0/)

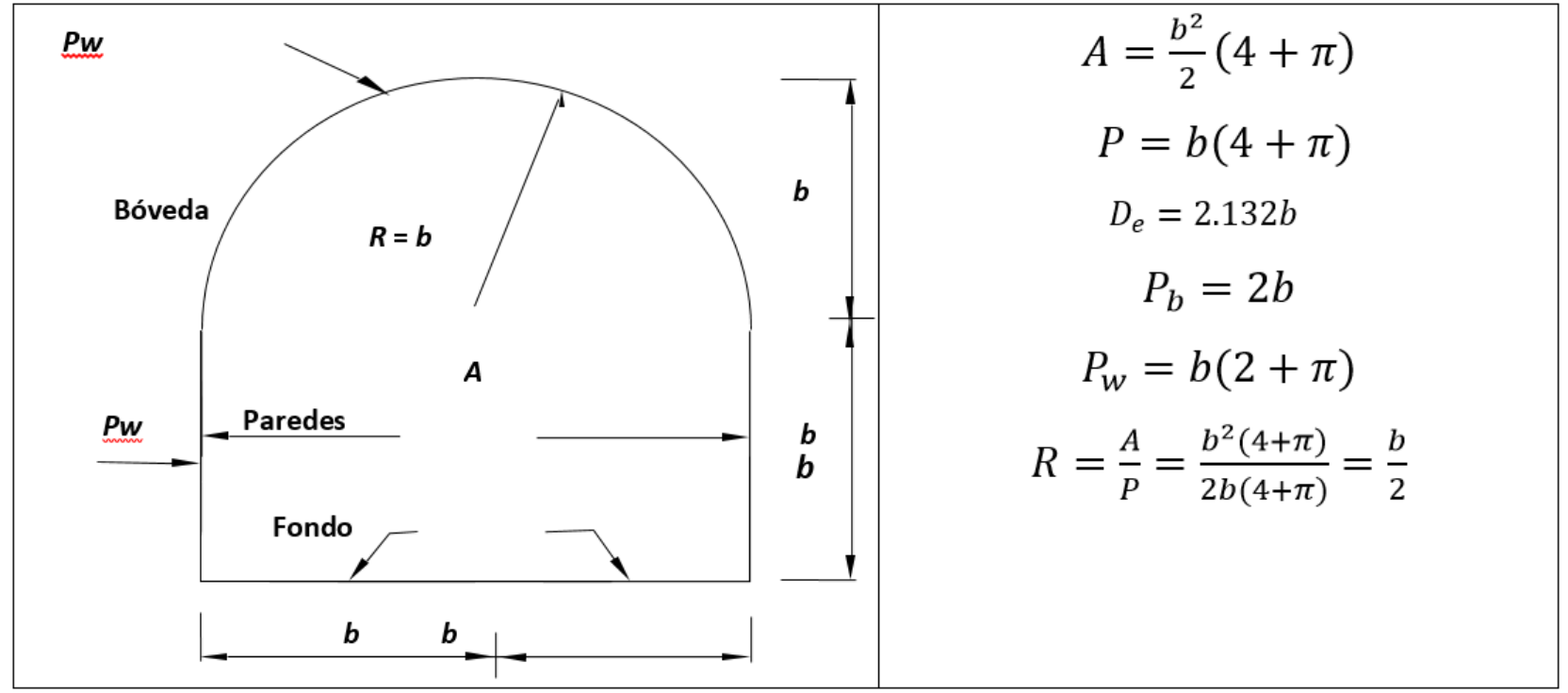

Figura 1. Sección geométrica baúl típica.

La Ecuación (7), con el criterio de Colebrook, queda:

$\frac{1}{\sqrt{\lambda}}=-2 \log \left(\frac{2 K c}{14.83 R}+\frac{(2.52) 2}{4 \frac{V b}{v} \sqrt{\lambda}}\right)$

La Ecuación (9) se emplea para el análisis experimental, donde $v$ es la viscosidad cinemática del flujo.

\section{Análisis de Aldama-Marengo}


Las hipótesis del planteamiento son las siguientes:

1. La pendiente de fricción se calcula con la expresión de Colebrook (Ecuación (9)), donde el diámetro es sustituido por cuatro veces el radio hidráulico (Yen, 2002). Para todas las secciones, incluyendo paredes y bóveda, se tiene:

$$
\begin{aligned}
& S_{\lambda}=\frac{\lambda V^{2}}{8 g R_{h}} \\
& S_{\lambda, b}=\frac{\lambda_{b} V^{2}}{8 g R_{h, b}} \\
& S_{\lambda, w}=\frac{\lambda_{w} V^{2}}{8 g R_{h, w}}
\end{aligned}
$$

2. Se considera que las pendientes de fricción en el fondo de la sección son iguales a las que se presentan en paredes y bóveda:

$$
S_{\lambda}=S_{\lambda, b}=S_{\lambda, w}=\frac{\lambda V^{2}}{R_{h}}=\frac{\lambda_{b} V^{2}}{R_{h, b}}=\frac{\lambda_{w} V^{2}}{R_{h, w}}
$$


3. Igualando las fuerzas, considerando que se tiene un flujo permanente y uniforme para la sección total del túnel, y sus zonas de influencia, se tiene que el esfuerzo cortante se expresa como:

$$
\begin{aligned}
& \tau=\rho g R_{h} S_{f} \\
& \tau_{b}=\rho g R_{h, b} S_{\lambda, b} \\
& \tau_{w}=\rho g R_{h, w} S_{\lambda, w}
\end{aligned}
$$

Combinando la Ecuación (14), Ecuación (15), Ecuación (16) y considerando la Ecuación (10), Ecuación (11) y Ecuación (12), se encuentra:

$$
\begin{gathered}
\tau=\rho \frac{\lambda V^{2}}{8} \\
\tau_{b}=\rho \frac{\lambda_{b} V_{b}^{2}}{8} \\
\tau_{w}=\rho \frac{\lambda_{w} V_{w}^{2}}{8}
\end{gathered}
$$


4. La fuerza cortante total (por unidad de longitud) que actúa sobre el perímetro mojado es igual a las fuerzas cortantes del fondo y el contorno:

$$
\lambda P V^{2}=\lambda_{b} P_{b} V_{b}^{2}+\lambda_{w} P_{w} V_{w}^{2}
$$

5. Aplicando la ecuación de Colebrook para toda la sección y las zonas de influencia del fondo y las paredes (incluyendo la bóveda) se obtiene:

$$
\begin{aligned}
& \frac{1}{\sqrt{\lambda}}=-2 \log \left(\frac{K P}{14.83 A}+\frac{v P}{1.59 V A \sqrt{\lambda}}\right) \\
& \frac{1}{\sqrt{\lambda_{b}}}=-2 \log \left(\frac{K_{b} P_{b}}{14.83 A_{b}}+\frac{P_{b} v}{1.59 A_{b} V_{b} \sqrt{\lambda_{b}}}\right) \\
& \frac{1}{\sqrt{\lambda_{w}}}=-2 \log \left(\frac{K_{w} P_{w}}{14.83 A_{w}}+\frac{P_{w} v}{1.59 A_{w} V_{w} \sqrt{\lambda_{w}}}\right)
\end{aligned}
$$

Nótese que esta hipótesis es aproximada, porque los coeficientes de la ecuación de Colebrook, que contienen los argumentos de las funciones logarítmicas en las ecuaciones anteriores, son estrictamente válidos para conductos de sección 
circular. No obstante, expresiones similares para canales anchos contienen variaciones no significativas para dichos coeficientes (Yen, 2002), por lo que se supondrá que el efecto dominante de la geometría se absorbe a través del radio hidráulico.

6. El área total de la sección es la suma de sus áreas parciales:

$$
A=A_{b}+A_{w}
$$

En este análisis se define:

$$
\alpha=\frac{A_{b}}{A} \Rightarrow 1-\alpha=\frac{A_{w}}{A}
$$

7. El perímetro mojado de la sección es la suma de los perímetros parciales del fondo y paredes:

$$
P=P_{b}+P_{w}
$$

También se define:

$$
\beta=\frac{P_{b}}{P} \Rightarrow 1-\beta=\frac{P_{w}}{P}
$$


8. El gasto total que pasa por la sección es la suma de los gastos del fondo y paredes:

$$
V A=V_{b} A_{b}+V_{w} A_{w}
$$

Esta hipótesis representa el principio de conservación de masa.

9. El factor de fricción asociado con la sección total es igual a los factores de fricción parciales asociados con el fondo y paredes:

$$
\lambda=\lambda_{b} \frac{P_{b}}{P}+\lambda_{w} \frac{P_{w}}{P}
$$

Esta expresión es similar a la propuesta por Yen (1992) para la ecuación de Manning.

A partir de la Ecuación (13) y combinándola con la Ecuación (21) se obtiene:

$$
V_{b}=\sqrt{\frac{A_{b}}{A} \frac{P}{P_{b}} \frac{\lambda}{\lambda_{b}}} V
$$


Tecnología y

Ciencias $₫$ Agua

$$
V_{w}=\sqrt{\frac{A_{w}}{A} \frac{P}{P_{w}} \frac{\lambda}{\lambda_{w}}} V
$$

Sustituyendo la Ecuación (30) y Ecuación (31) en la Ecuación (20) se obtiene:

$$
P=\frac{A_{b} / P_{b}}{A} P P_{b}+\frac{A_{w} / P_{w}}{A} P P_{w}
$$

Sustituyendo la Ecuación (25), Ecuación (27), Ecuación (30) y Ecuación (31) en la Ecuación (22) y Ecuación (23), y simplificando se llega a:

$$
\begin{aligned}
& \frac{1}{\sqrt{\lambda_{b}}}=-2 \log \left(\frac{v \beta^{3 / 2} P}{1.59 V \alpha^{3 / 2} A \sqrt{\lambda}}+\frac{K_{b} \beta P}{14.83 \alpha A}\right) \\
& \frac{1}{\sqrt{\lambda_{w}}}=-2 \log \left(\frac{v(1-\beta)^{3 / 2} P}{1.59 V(1-\alpha)^{3 / 2} A \sqrt{\lambda}}+\frac{K_{w}(1-\beta) P}{14.83(1-\alpha) A}\right)
\end{aligned}
$$

Si la Ecuación (25), Ecuación (27), Ecuación (22) y Ecuación (23) se sustituyen en la Ecuación (28) se tiene:

$$
\frac{1}{\sqrt{\lambda}}=\frac{\alpha^{1 / 2}}{\beta^{1 / 2}} \frac{1}{\lambda_{b}^{1 / 2}} \alpha+\frac{(1-\alpha)^{1 / 2}}{(1-\beta)^{1 / 2}} \frac{\lambda^{1 / 2}}{\lambda_{w}^{1 / 2}}(1-\alpha)
$$


Tecnología y

Ciencias $₫$ Agua
2020, Instituto Mexicano de Tecnología del Agua

Open Access bajo la licencia CC BY-NC-SA 4.0

(https://creativecommons.org/licenses/by-nc-sa/4.0/)

Sustituyendo entonces la Ecuación (33) y Ecuación (34) en la Ecuación (35):

$$
\begin{aligned}
& \lambda=\left\langle\frac{\alpha^{3 / 2}}{\beta^{1 / 2}}\left[-2 \log \left(\frac{v \beta^{3 / 2} P}{1.59 V \alpha^{3 / 2} A \sqrt{\lambda}}+\frac{K_{b} \beta P}{14.83 \alpha A}\right)\right]+\right. \\
& \left.\frac{(1-\alpha)^{3 / 2}}{(1-\beta)^{1 / 2}}\left\{-2 \log \left(\frac{v(1-\beta)^{3 / 2} P}{1.59 V(1-\alpha)^{3 / 2} A \sqrt{\lambda}}+\frac{K_{W}(1-\beta) P}{14.83(1-\alpha) A}\right)\right\}\right\rangle^{-2}
\end{aligned}
$$

Sustituyendo la Ecuación (33) y Ecuación (34) en la Ecuación (29):

$$
\begin{aligned}
& \lambda=\frac{1}{4} \beta\left[\log \left(\frac{v \beta^{3 / 2} P}{1.59 V \alpha^{3 / 2} A \sqrt{\lambda}}+\frac{K_{b} \beta P}{14.83 \alpha A}\right)\right]^{-2}+\frac{1}{4}(1- \\
& \beta)\left[\log \left(\frac{v(1-\beta)^{3 / 2} P}{1.59 V(1-\alpha)^{3 / 2} A \sqrt{\lambda}}+\frac{K_{W}(1-\beta) P}{14.83(1-\alpha) A}\right)\right]^{-2}
\end{aligned}
$$

Igualando la Ecuación (36) y Ecuación (37):

$$
\begin{aligned}
& \frac{1}{4} \beta\left[\log \left(\frac{v \beta^{3 / 2} P}{1.59 V \alpha^{3 / 2} A \sqrt{\lambda}}+\frac{K_{b} \beta P}{14.83 \alpha A}\right)\right]^{-2}+\frac{1}{4}(1-\beta)\left[\operatorname { l o g } \left(\frac{v(1-\beta)^{3 / 2} P}{1.59 V(1-\alpha)^{3 / 2} A \sqrt{\lambda}}+\right.\right. \\
& \left.\left.\frac{K_{W}(1-\beta) P}{14.83(1-\alpha) A}\right)\right]^{-2}=\left\langle\left\langle\frac{\alpha^{3 / 2}}{\beta^{1 / 2}}\left[-2 \log \left(\frac{v \beta^{3 / 2} P}{1.59 V \alpha^{3 / 2} A \sqrt{\lambda}}+\frac{K_{b} \beta P}{14.83 \alpha A}\right)\right]+\right.\right. \\
& \left.\frac{(1-\alpha)^{3 / 2}}{(1-\beta)^{1 / 2}}\left\{-2 \log \left(\frac{v(1-\beta)^{3 / 2} P}{1.59 V(1-\alpha)^{3 / 2} A \sqrt{\lambda}}+\frac{K_{W}(1-\beta) P}{14.83(1-\alpha) A}\right)\right\}\right\rangle^{-2}
\end{aligned}
$$


Sólo se tiene como incógnita el valor de $\alpha$ y se puede resolver con algún método numérico. De hecho, se presentan dos casos de análisis:

Caso I. Si se conoce el factor de rugosidad $\lambda$ de manera experimental y además se sabe la rugosidad del fondo $K_{b}$, entonces se pueden calcular la rugosidad de las paredes y bóveda $K_{w}$. En este caso, también son incógnitas $\lambda_{w}, \lambda_{b}, V_{b}, V_{w}, A_{b}, A_{w}, K_{w}$ y $\alpha$.

Caso II. Para un análisis específico se conocen la rugosidad absoluta del piso y paredes $\left(K_{b}, K_{w}\right)$ y se determinan los perímetros mojados $\left(P_{b}, P_{w}\right)$ a partir de la geometría de los túneles. En esta situación, las incógnitas son $\lambda, \lambda_{b}, V_{b}, V_{w}, A_{b}, A_{w}, K_{w}, P_{w}$ y $\alpha$.

\section{Modelos hidráulicos con un material}

Los autores de este artículo (Marengo, 2016) hicieron una experimentación con modelos que se construyeron para analizar el comportamiento del flujo de agua en túneles con rugosidad compuesta. Los materiales utilizados fueron acrílico, lija, plástico y carpeta. 
El aparato experimental empleado para la modelación se muestra en la Figura 2. Los ensayes se hicieron con túneles que tienen un ancho de sección $2 b=0.133 \mathrm{~m}\left(D_{e}=0.14179 \mathrm{~m}\right)$; la pendiente es variable con 9 $\mathrm{m}$ de longitud. Las aristas de entrada y salida fueron redondeadas. Se modelaron diferentes gastos con cada uno de los materiales señalados, con el objeto de conocer sus propiedades hidráulicas en cada uno de ellos. Posteriormente se hizo la modelación con materiales combinados, utilizando el acrílico como material de fondo, y lija, plástico y carpeta en paredes y bóveda en cada uno de los túneles ensayados. En el análisis se consideró el criterio de similitud de Froude.

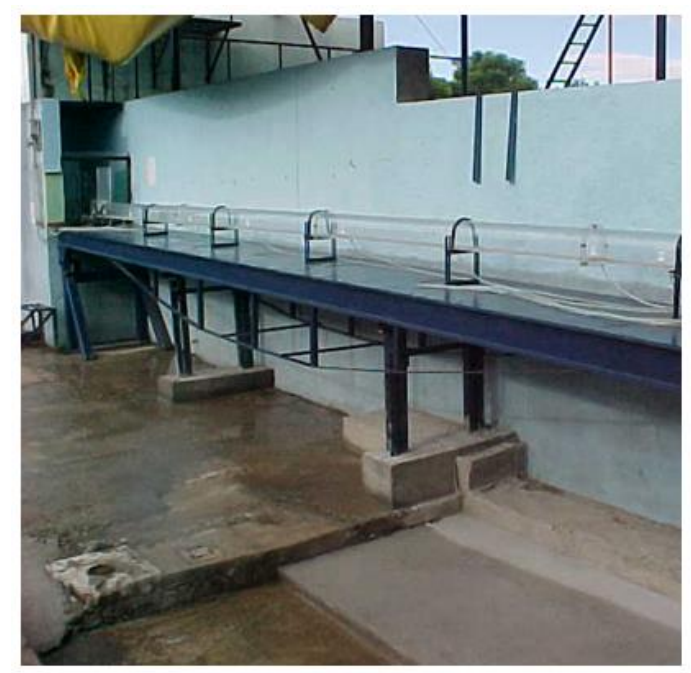

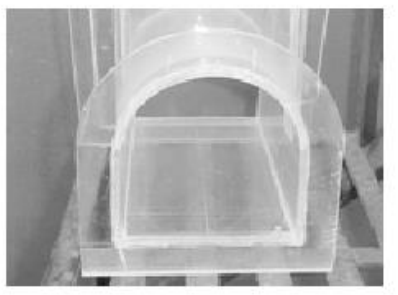

Acrílico

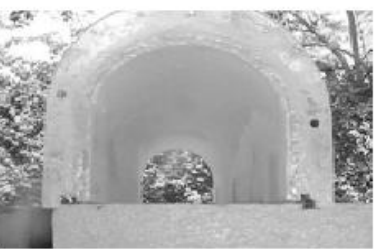

Plástico

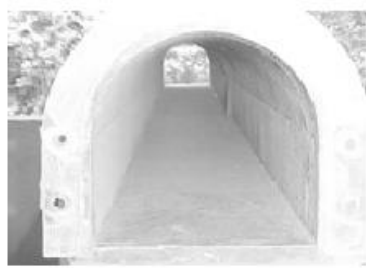

Lija

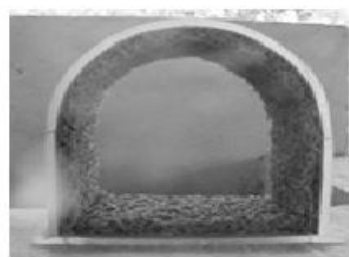

Carpeta

Figura 2. Aparato experimental y materiales utilizados.

Para determinar el gradiente hidráulico entre dos secciones de los túneles estudiados se usaron como mediciones del fenómeno físico las 
Tecnología y

Ciencias $₫$ Agua
2020, Instituto Mexicano de Tecnología del Agua

Open Access bajo la licencia CCBY-NC-SA 4.0

(https://creativecommons.org/licenses/by-nc-sa/4.0/)

cargas piezométricas en las secciones a distancia $6 D_{e}$ y $28 D_{e}$ de los túneles modelados ( $\left.L_{1-2}=3119 \mathrm{~m}\right)$. De acuerdo con la geometría de las aristas de la entrada de los túneles hay un fuerte efecto de la mezcla agua-aire aguas arriba de la sección $6 D_{e}$, así como aguas abajo de la sección $28 D_{e}$ por lo que los estudios como tubo lleno se ubicaron en este tramo. Como mediciones propias del fenómeno físico, sólo se utilizaron las cargas piezométricas ya indicadas.

Los datos de entrada del modelo son los flujos asociados con cada una de las pendientes, las cargas piezométricas medidas y las rugosidades obtenidas para los modelos construidos con un solo material (Tabla 1), donde se indica el coeficiente de rugosidad $K_{C b}$ encontrado con el criterio de Colebrook, que se considera como valor verdadero en el estudio, y los obtenidos con las expresiones de Nikuradse (1933), Haland (1983) Churchill (1973) y Swamee (1976).

Tabla 1. Resultados de modelos con acrílico.

\begin{tabular}{|c|c|c|c|c|c|c|c|c|l|}
\hline $\boldsymbol{s}$ & $\begin{array}{c}Q \\
\left(\mathrm{~m}^{3} / \mathrm{s}\right)\end{array}$ & $V(\mathrm{~m} / \mathrm{s})$ & 4Re & $\lambda$ medido & Nikuradse & Haaland & Churchil & Swamee & $K_{C b}$ medido \\
\hline \multirow{4}{*}{$\begin{array}{c}\text { A1 } \\
\mathbf{0 . 0 0 0 7}\end{array}$} & 0.0214 & 1.355 & 180,648 & 0.01787 & 0.000090 & 0.000044 & 0.000075 & 0.000037 & 0.0000431 \\
\cline { 2 - 9 } & 0.0228 & 1.444 & 192,467 & 0.01785 & 0.000089 & 0.000046 & 0.000075 & 0.000040 & 0.0000456 \\
\cline { 2 - 10 } & 0.0245 & 1.552 & 206,817 & 0.01698 & 0.000072 & 0.000030 & 0.000058 & 0.000025 & 0.0000296 \\
\cline { 2 - 9 } & 0.0258 & 1.634 & 217,791 & 0.01766 & 0.000085 & 0.000047 & 0.000072 & 0.000041 & 0.0000469 \\
\cline { 2 - 9 } & 0.0196 & 1.241 & 165,454 & 0.01762 & 0.000084 & 0.000034 & 0.000068 & 0.000028 & 0.0000328 \\
\hline & 0.0214 & 1.355 & 180,648 & 0.01697 & 0.000072 & 0.000024 & 0.000057 & 0.000019 & 0.0000316 \\
\hline
\end{tabular}


Teçnología y

Ciencias $₫$ Agua
2020, Instituto Mexicano de Tecnología del Agua

Open Access bajo la licencia CC BY-NC-SA 4.0

(https://creativecommons.org/licenses/by-nc-sa/4.0/)

\begin{tabular}{|c|l|l|l|l|l|l|l|l|l|}
\hline \multirow{4}{*}{$\begin{array}{c}\text { A2 } \\
\mathbf{0 . 0 0 1}\end{array}$} & 0.0229 & 1.450 & 193,311 & 0.01735 & 0.000079 & 0.000035 & 0.000065 & 0.000029 & 0.0000426 \\
\cline { 2 - 9 } & 0.0245 & 1.552 & 206,817 & 0.01664 & 0.000065 & 0.000024 & 0.000052 & 0.000019 & 0.0000289 \\
\cline { 2 - 9 } & 0.0257 & 1.628 & 216,947 & 0.01744 & 0.000081 & 0.000042 & 0.000068 & 0.000036 & 0.0000482 \\
\hline \multirow{4}{*}{$\begin{array}{c}\text { A3 } \\
\mathbf{0 . 0 0 4}\end{array}$} & 0.0228 & 1.444 & 191,102 & 0.01763 & 0.000081 & 0.000037 & 0.000066 & 0.000031 & 0.0000357 \\
\cline { 2 - 9 } & 0.0261 & 1.653 & 218,761 & 0.01762 & 0.000080 & 0.000042 & 0.000068 & 0.000036 & 0.0000416 \\
\cline { 2 - 9 } & 0.0275 & 1.741 & 230,495 & 0.01745 & 0.000077 & 0.000041 & 0.000065 & 0.000035 & 0.0000402 \\
\hline
\end{tabular}

Se reportan para las pendientes probadas A1-0.0007, A2-0.001 y A3-0.004; para cada uno de los gastos estudiados, la velocidad media $V$ $=Q / A$, y el número de Reynolds utilizado $(4 R e)$, que considera un valor de la viscosidad cinemática del agua con valor $v=0.000001 \mathrm{~m}^{2} / \mathrm{s}$; se obtiene el valor del coeficiente de rugosidad $K_{b}$ para cada criterio y, como se señaló, el de Colebrook-White, el que se usa en la modelación teórica de la rugosidad compuesta para el material del fondo.

En la Tabla 2 se muestran los parámetros hidráulicos para la lija, con pendientes L1-0.001, L2-0.004 y L3-0.008. En cada caso se estimó la rugosidad absoluta evaluada con la ecuación de Colebrook-White $K_{C b}$, que se reporta como valor medido.

Tabla 2. Resultados de modelos con lija.

\begin{tabular}{|c|c|c|c|c|c|c|c|}
\hline$S$ & $\begin{array}{c}Q \\
\left(\mathrm{~m}^{3} / \mathrm{s}\right)\end{array}$ & $V(\mathrm{~m} / \mathrm{s})$ & $4 R e$ & $\Delta h$ & $I$ & $\lambda_{\text {medido }}$ & $\begin{array}{c}K_{C b} \\
\text { medido }\end{array}$ \\
\hline
\end{tabular}


Tecnología y

Ciencias Agua
2020, Instituto Mexicano de Tecnología del Agua

Open Access bajo la licencia CC BY-NC-SA 4.0

(https://creativecommons.org/licenses/by-nc-sa/4.0/)

\begin{tabular}{|c|c|c|c|c|c|c|c|}
\hline \multirow{5}{*}{$\begin{array}{c}\text { L1 } \\
0.001\end{array}$} & 0.0170 & 1.0766 & 143183 & 0.0457 & 0.0146 & 0.03294 & 0.000819 \\
\hline & 0.0190 & 1.2032 & 160028 & 0.0544 & 0.0174 & 0.03136 & 0.000697 \\
\hline & 0.0200 & 1.2665 & 168450 & 0.0649 & 0.0208 & 0.03378 & 0.000898 \\
\hline & 0.0210 & 1.3299 & 176873 & 0.0720 & 0.0230 & 0.03399 & 0.000919 \\
\hline & 0.0230 & 1.4565 & 193718 & 0.0816 & 0.0261 & 0.03208 & 0.000761 \\
\hline \multirow{4}{*}{$\begin{array}{c}L 2 \\
0.004\end{array}$} & 0.0190 & 1.2032 & 160028 & 0.0603 & 0.0193 & 0.03149 & 0.000982 \\
\hline & 0.0210 & 1.3299 & 176873 & 0.0731 & 0.0234 & 0.03473 & 0.000962 \\
\hline & 0.0220 & 1.3932 & 185295 & 0.0804 & 0.0257 & 0.03447 & 0.000974 \\
\hline & 0.0240 & 1.5199 & 202140 & 0.0927 & 0.0296 & 0.03458 & 0.000879 \\
\hline \multirow{5}{*}{$\begin{array}{c}\text { L3 } \\
0.008\end{array}$} & 0.0180 & 1.1399 & 151605 & 0.0570 & 0.0182 & 0.0366 & 0.001157 \\
\hline & 0.0200 & 1.2665 & 168450 & 0.0665 & 0.0213 & 0.0346 & 0.000971 \\
\hline & 0.0210 & 1.3299 & 176873 & 0.0777 & 0.0249 & 0.0367 & 0.001172 \\
\hline & 0.0230 & 1.4565 & 193718 & 0.0837 & 0.0268 & 0.0329 & 0.000830 \\
\hline & 0.0240 & 1.5199 & 202140 & 0.0944 & 0.0302 & 0.0371 & 0.001215 \\
\hline Media & & & & & & & 0.000885 \\
\hline
\end{tabular}

La Tabla 3 muestra los parámetros hidráulicos para el plástico con pendientes L1-0.001, L2-0.004 y L3-0.008. En cada caso se estimó la rugosidad absoluta evaluada con la ecuación de Colebrook.

Tabla 3. Resultados de modelos con plástico.

\begin{tabular}{|c|c|c|c|c|c|c|c|}
\hline$S$ & $\begin{array}{c}Q \\
\left(\mathrm{~m}^{3} / \mathrm{s}\right)\end{array}$ & $V(\mathrm{~m} / \mathrm{s})$ & $4 R e$ & $\Delta h$ & $I$ & $\lambda_{\text {medido }}$ & $\begin{array}{c}K c b \\
\text { medido }\end{array}$ \\
\hline
\end{tabular}


Tecnología y

Ciencias $\approx$ Agua
2020, Instituto Mexicano de Tecnología del Agua

Open Access bajo la licencia CC BY-NC-SA 4.0

(https://creativecommons.org/licenses/by-nc-sa/4.0/)

\begin{tabular}{|c|c|c|c|c|c|c|c|}
\hline \multirow{5}{*}{$\begin{array}{c}\text { P2 } \\
0.001\end{array}$} & 0.015 & 0.950 & 126338 & 0.0420 & 0.01345 & 0.04156 & 0.001739 \\
\hline & 0.017 & 1.077 & 143183 & 0.0511 & 0.01636 & 0.03935 & 0.001488 \\
\hline & 0.019 & 1.203 & 160028 & 0.0612 & 0.01958 & 0.03772 & 0.001314 \\
\hline & 0.020 & 1.267 & 168450 & 0.0709 & 0.02267 & 0.03942 & 0.001495 \\
\hline & 0.021 & 1.330 & 176873 & 0.0420 & 0.02590 & 0.04084 & 0.001655 \\
\hline \multirow{4}{*}{$\begin{array}{c}\text { P3 } \\
0.004\end{array}$} & 0.018 & 1.140 & 151605 & 0.0608 & 0.01468 & 0.03907 & 0.001456 \\
\hline & 0.019 & 1.203 & 160028 & 0.0698 & 0.01945 & 0.04022 & 0.001584 \\
\hline & 0.021 & 1.330 & 176873 & 0.0799 & 0.02232 & 0.03769 & 0.001310 \\
\hline & 0.022 & 1.393 & 185295 & 0.0868 & 0.02554 & 0.03733 & 0.001274 \\
\hline \multirow{5}{*}{$\begin{array}{c}\text { P4 } \\
0.008\end{array}$} & 0.016 & 1.013 & 134760 & 0.0490 & 0.01568 & 0.03985 & 0.001542 \\
\hline & 0.018 & 1.140 & 151605 & 0.0597 & 0.01909 & 0.03833 & 0.001377 \\
\hline & 0.019 & 1.203 & 160028 & 0.0688 & 0.02200 & 0.03965 & 0.001520 \\
\hline & 0.020 & 1.267 & 168450 & 0.0880 & 0.02813 & 0.04576 & 0.002268 \\
\hline & 0.022 & 1.393 & 185295 & 0.0959 & 0.03068 & 0.04124 & 0.001702 \\
\hline Media & & & & & & & 0.001448 \\
\hline
\end{tabular}

La Tabla 4 muestra finalmente los parámetros hidráulicos para la carpeta con pendientes C1-0.001, C2-0.004 y C3-0.008.

Tabla 4. Resultados de modelos con carpeta.

\begin{tabular}{|c|c|c|c|c|c|c|c|}
\hline $\boldsymbol{S}$ & $Q\left(\mathrm{~m}^{3} / \mathrm{s}\right)$ & $V(\mathrm{~m} / \mathrm{s})$ & $4 R e$ & $\Delta h$ & $I$ & $\lambda_{\text {medido }}$ & $\begin{array}{c}\boldsymbol{K}_{\boldsymbol{C b}} \\
\text { medido }\end{array}$ \\
\hline $\mathrm{C2} \mathbf{0 . 0 0 1}$ & 0.0097 & 0.614 & 81698 & 0.041 & 0.01326 & 0.09173 & 0.011017 \\
\hline
\end{tabular}


Tecnología y

Ciencias $\approx$ Agua
2020, Instituto Mexicano de Tecnología del Agua

Open Access bajo la licencia CC BY-NC-SA 4.0

(https://creativecommons.org/licenses/by-nc-sa/4.0/)

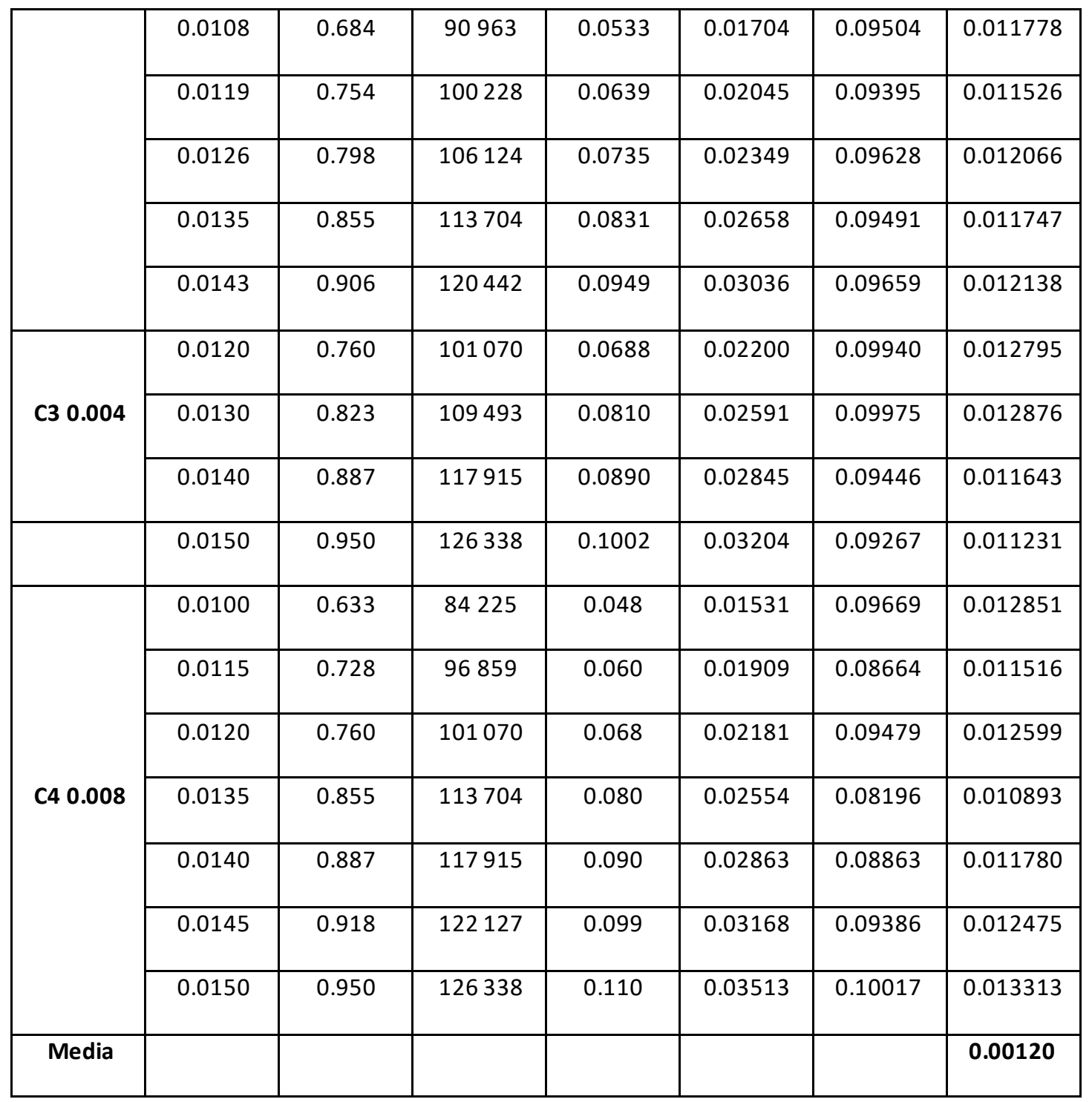

Para este análisis, el número de Reynolds se reduce en comparación con los otros modelos estudiados, pero el efecto de la rugosidad es significativo y en el Ábaco de Moody estos modelos caen fuera de la zona de transición. 


\section{Aplicación y validación del nuevo criterio (Aldama-Marengo-Romero)}

Los resultados experimentales del modelo acrílico-lija se muestran en el Anexo I, donde se pueden observar en las tablas correspondientes los resultados de las pruebas de modelos hechas para cada material y para las combinaciones acrílico-lija, acrílico-plástico y acrílico-carpeta con pendientes $S 1=0.001, S 2=0.004, S 3=0.008$.

De las tablas elaboradas para el caso de acrílico-lija se obtuvieron los resultados de la rugosidad absoluta que, en cada caso como era de esperarse, muestran valores menores a los obtenidos sólo con el material de lija.

Los valores del número de Reynolds oscilan entre 140000 y 203 000, cayendo claramente en la zona de transición. Se aplica entonces el criterio para el Caso I, y se obtiene el valor $K_{c w}$ medio, que es el de la rugosidad absoluta, calculado en paredes y bóveda.

Para validar los modelos se sigue lo planteado para el Caso II, comentado líneas arriba. Se parte de conocer que en el piso $K b=$ 0.000387 (promedio del criterio de Colebrook obtenido para el acrílico), y se trata de conocer o suponer las magnitudes de la rugosidad absoluta $K_{w}$, calculado de las paredes y bóveda del túnel analizado, lo que se hace 
Tecnología y

Ciencias $₫$ Agua
2020, Instituto Mexicano de Tecnología del Agua

Open Access bajo la licencia CC BY-NC-SA 4.0

(https://creativecommons.org/licenses/by-nc-sa/4.0/)

con los valores del coeficiente de rugosidad previamente calculados con el Caso I; entonces se pueden estimar los parámetros $\lambda, \lambda_{b}, V_{b}, V_{w}, A_{b}, A_{w}$, $P_{w}$ y $\alpha$, lo que permite conocer el valor de $K$ medio validado, que se muestra para fines comparativos en la Tabla 5.

Tabla 5. Resultados de los modelos experimentales acrílico-lija.

\begin{tabular}{|c|c|c|c|c|c|c|}
\hline$S$ & $Q\left(\mathrm{~m}^{3} / \mathrm{s}\right)$ & $\lambda$ medido & $\begin{array}{c}K C w \\
\text { medido en } \\
\text { modelo }\end{array}$ & $K c w$ calculado & $\lambda($ validado $)$ & $\begin{array}{l}K_{c} \text { medio } \\
\text { (validado) }\end{array}$ \\
\hline \multirow{5}{*}{$\begin{array}{c}\text { AL1 } \\
0.001\end{array}$} & 0.01700 & 0.02716 & 0.000404 & 0.000697 & 0.02720 & 0.000423 \\
\hline & 0.01900 & 0.02730 & 0.000418 & 0.000691 & 0.02732 & 0.000417 \\
\hline & 0.02000 & 0.02737 & 0.000424 & 0.000706 & 0.02737 & 0.000424 \\
\hline & 0.02200 & 0.02719 & 0.000417 & 0.000697 & 0.02712 & 0.000417 \\
\hline & 0.02300 & 0.02884 & 0.000523 & 0.000904 & 0.02884 & 0.000523 \\
\hline \multirow{5}{*}{$\begin{array}{c}\text { AL2 } \\
0.004\end{array}$} & 0.01800 & 0.02943 & 0.000553 & 0.000948 & 0.02943 & 0.000553 \\
\hline & 0.02000 & 0.02900 & 0.000528 & 0.000907 & 0.02906 & 0.000528 \\
\hline & 0.02100 & 0.02858 & 0.000502 & 0.000859 & 0.02858 & 0.000502 \\
\hline & 0.02200 & 0.02976 & 0.000584 & 0.001020 & 0.02974 & 0.000583 \\
\hline & 0.02400 & 0.02788 & 0.000462 & 0.000788 & 0.02787 & 0.000462 \\
\hline \multirow{5}{*}{$\begin{array}{l}\text { AL3 } \\
0.008\end{array}$} & 0.01800 & 0.03346 & 0.000866 & 0.001326 & 0.03202 & 0.000745 \\
\hline & 0.02000 & 0.03072 & 0.000650 & 0.001460 & 0.03271 & 0.000807 \\
\hline & 0.02100 & 0.03214 & 0.000773 & 0.001375 & 0.03214 & 0.000773 \\
\hline & 0.02300 & 0.03299 & 0.000836 & 0.001530 & 0.03298 & 0.000835 \\
\hline & 0.02400 & 0.03234 & 0.000784 & 0.001429 & 0.03234 & 0.000784 \\
\hline
\end{tabular}


Tecnología y

Ciencias $\approx$ Agua
2020, Instituto Mexicano de Tecnología del Agua

Open Access bajo la licencia CC BY-NC-SA 4.0

(https://creativecommons.org/licenses/by-nc-sa/4.0/)

Puede observarse que se obtiene una buena correlación entre los resultados obtenidos para el $K_{c} w$ medido en el modelo y el $K_{c w}$ medio validado.

Los resultados conseguidos permiten concluir que el modelo teórico es enteramente aplicable a un caso práctico. Se sigue el mismo procedimiento para la combinación acrílico-plástico, que permite comparar los valores medios y los validados, como se muestra en la Tabla 6.

Tabla 6. Resultados de los modelos experimentales acrílico-plástico.

\begin{tabular}{|c|c|c|c|c|c|c|}
\hline$s$ & $\begin{array}{c}Q \\
\left(\mathrm{~m}^{3} / \mathrm{s}\right)\end{array}$ & $\lambda$ medido & $\begin{array}{c}K_{C w} \text { medido } \\
\text { en modelo }\end{array}$ & $K_{C W}$ calculado & $\lambda($ validado $)$ & $\begin{array}{l}\boldsymbol{K}_{\boldsymbol{C}} \text { medio } \\
\text { (validado) }\end{array}$ \\
\hline \multirow{5}{*}{$\begin{array}{l}\text { AP1 } \\
0.001\end{array}$} & 0.0160 & 0.033013 & 0.000822 & 0.001520 & 0.033309 & 0.000847 \\
\hline & 0.0170 & 0.035227 & 0.001023 & 0.001880 & 0.035233 & 0.001023 \\
\hline & 0.0190 & 0.034086 & 0.000923 & 0.001690 & 0.034086 & 0.000923 \\
\hline & 0.0200 & 0.035409 & 0.001047 & 0.001947 & 0.035411 & 0.001047 \\
\hline & 0.0210 & 0.035864 & 0.001092 & 0.002045 & 0.035865 & 0.001092 \\
\hline \multirow{5}{*}{$\begin{array}{l}\text { AP2 } \\
0.004\end{array}$} & 0.0170 & 0.035550 & 0.001053 & 0.001940 & 0.035547 & 0.001053 \\
\hline & 0.0180 & 0.036900 & 0.001188 & 0.002220 & 0.03691 & 0.00187 \\
\hline & 0.0200 & 0.034315 & 0.000946 & 0.001741 & 0.03431 & 0.000946 \\
\hline & 0.0210 & 0.034604 & 0.000974 & 0.001841 & 0.034806 & 0.000992 \\
\hline & 0.0230 & 0.038385 & 0.001350 & 0.002591 & 0.038387 & 0.00135 \\
\hline \multirow{2}{*}{$\begin{array}{l}\text { AP3 } \\
0.008\end{array}$} & 0.0180 & 0.035010 & 0.001005 & 0.001850 & 0.035013 & 0.001005 \\
\hline & 0.0200 & 0.034333 & 0.000948 & 0.001744 & 0.034331 & 0.000947 \\
\hline
\end{tabular}




\begin{tabular}{|l|l|l|l|l|l|l|}
\hline & 0.0210 & 0.035690 & 0.001075 & 0.002010 & 0.035656 & 0.001075 \\
\cline { 2 - 7 } & 0.0220 & 0.035933 & 0.001100 & 0.002067 & 0.035931 & 0.00110 \\
\cline { 2 - 7 } & 0.0240 & 0.035859 & 0.001096 & 0.002070 & 0.035866 & 0.001097 \\
\hline
\end{tabular}

Para la pendiente AP-001, a partir del gasto $Q=0.020 \mathrm{~m}^{3} / \mathrm{s}, V=$ $1.266 \mathrm{~m} / \mathrm{s}, 4 R e=168450, \lambda=0.035409$. Considerando en la plantilla una rugosidad con valor $K_{b}=0.0000387 \mathrm{~mm}$ se obtiene en el análisis un valor $\alpha=0.160$, para el cual $A_{b}=0.0025, A_{w}=0.0133, \lambda_{b}=0.02049, \lambda_{w}$ $=0.04126, \mathrm{y}$ el resultado de la rugosidad para paredes y bóveda es $K_{w}=$ 0.001947 .

Debe señalarse que en los modelos estudiados con el material sólo del acrílico para un gasto similar, el coeficiente de rugosidad es de $\lambda=$ 0.0177; el incremento a un valor $\lambda_{b}=0.02049$ puede explicarse por la influencia de la rugosidad en las paredes y bóveda. Puede observarse que se obtiene una muy buena correlación entre $K_{c} w$ medido en el modelo y el $K_{c w}$ medio validado.

En la Tabla 7 se sigue el mismo procedimiento para los modelos acrílico-carpeta; es necesario notar que debido a la fuerte rugosidad que produce este material (carpeta), los números de Reynolds son uniformemente altos y el flujo se encuentra en una zona de turbulencia desarrollada por completo, a diferencia de los modelos anteriores, en los que se encuentran en zonas de transición e inicio de turbulencia desarrollada.

Tabla 7. Resultados de los modelos experimentales acrílico-carpeta. 
Tecnología y

Ciencias $₫$ Agua
2020, Instituto Mexicano de Tecnología del Agua

Open Access bajo la licencia CC BY-NC-SA 4.0

(https://creativecommons.org/licenses/by-nc-sa/4.0/)

\begin{tabular}{|c|c|c|c|c|c|c|c|}
\hline$s$ & $Q\left(\mathrm{~m}^{3} / \mathrm{s}\right)$ & $4 R e$ & $\lambda$ medido & $K_{C w}$ medido & $\begin{array}{c}\text { KCw } \\
\text { calculado }\end{array}$ & $\begin{array}{c}\lambda \text { medido } \\
\text { validado }\end{array}$ & $\begin{array}{l}K_{c} \text { medio } \\
\text { (validado) }\end{array}$ \\
\hline \multirow{5}{*}{$\begin{array}{l}\text { AC1 } \\
0.001\end{array}$} & 0.0124 & 104439 & 0.06909 & 0.00613 & 0.012240 & 0.06909 & 0.00613 \\
\hline & 0.0136 & 114546 & 0.07099 & 0.00651 & 0.013049 & 0.07099 & 0.00651 \\
\hline & 0.0145 & 122127 & 0.07158 & 0.00663 & 0.013320 & 0.07158 & 0.00663 \\
\hline & 0.0154 & 129707 & 0.07142 & 0.00660 & 0.01329 & 0.07142 & 0.00660 \\
\hline & 0.0163 & 137287 & 0.07086 & 0.00649 & 0.013093 & 0.07086 & 0.00649 \\
\hline \multirow{5}{*}{$\begin{array}{l}A C 2 \\
0.004\end{array}$} & 0.0110 & 92648 & 0.06955 & 0.00621 & 0.012351 & 0.06955 & 0.00621 \\
\hline & 0.0120 & 101070 & 0.07237 & 0.00678 & 0.013533 & 0.07237 & 0.00678 \\
\hline & 0.0130 & 109493 & 0.07458 & 0.00724 & 0.01448 & 0.07458 & 0.00724 \\
\hline & 0.0140 & 117915 & 0.07123 & 0.00656 & 0.01316 & 0.07123 & 0.00656 \\
\hline & 0.0150 & 126338 & 0.07084 & 0.00648 & 0.013042 & 0.07084 & 0.00648 \\
\hline \multirow{5}{*}{$\begin{array}{l}\text { AC3 } \\
0.008\end{array}$} & 0.0130 & 109493 & 0.07200 & 0.00671 & 0.013428 & 0.07200 & 0.00671 \\
\hline & 0.0140 & 117915 & 0.07232 & 0.00678 & 0.013603 & 0.07232 & 0.00678 \\
\hline & 0.0150 & 126338 & 0.07283 & 0.00689 & 0.013848 & 0.07283 & 0.00689 \\
\hline & 0.0160 & 134760 & 0.07266 & 0.00685 & 0.013815 & 0.07266 & 0.00685 \\
\hline & 0.0170 & 143183 & 0.07161 & 0.006643 & 0.013417 & 0.07161 & 0.006643 \\
\hline
\end{tabular}

Cabe señalar que en los modelos estudiados con el material sólo del acrílico, el coeficiente de rugosidad es de $\lambda=0.0177$; el incremento a un valor $\lambda_{b}=0.02049$ puede explicarse por la influencia de la rugosidad en las paredes y bóveda.

Para la aplicación del Caso II, como se hizo para el caso anterior, se parte del valor del coeficiente de rugosidad en el piso $(K b=0.000387)$, 
y se utilizan para su validación las magnitudes de la rugosidad absoluta $K_{w}$ de las paredes y bóveda del túnel analizado con los valores de del coeficiente de rugosidad estimados con el Caso I.

En el Anexo I se muestran las tablas de cálculo que permiten obtener los resultados mostrados en la Tabla 5, Tabla 6 y Tabla 7.

Para visualizar los resultados obtenidos se preparó la Figura 3, que es el Ábaco de Moody, con un rango del número de Reynolds entre 60 000 y 600000 se redujo a 240000 donde se muestran los cálculos anteriores. 
Tecnología y

Ciencias $₫$ Agua
2020, Instituto Mexicano de Tecnología del Agua

Open Access bajo la licencia CC BY-NC-SA 4.0

(https://creativecommons.org/licenses/by-nc-sa/4.0/)

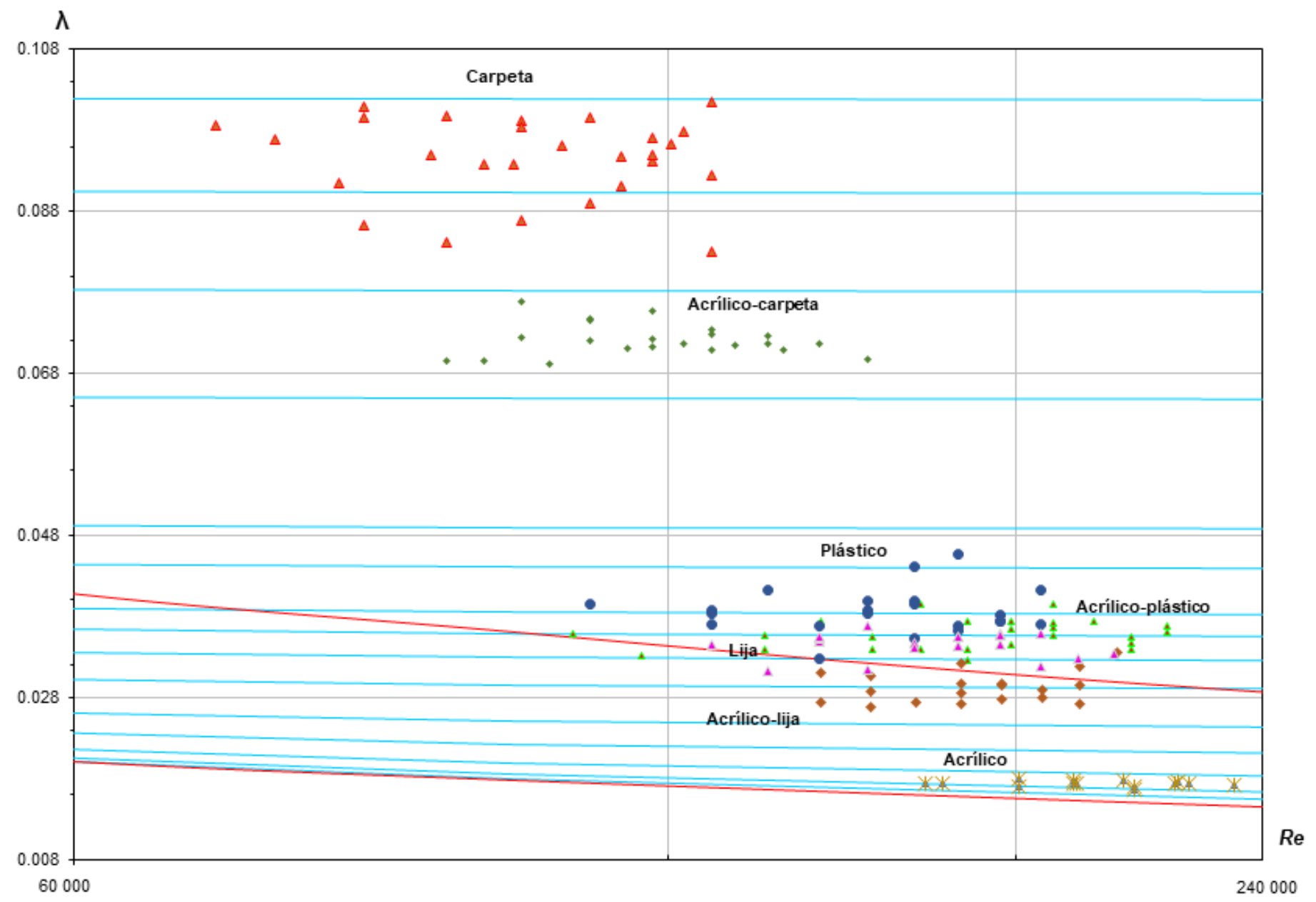

Figura 3. Ábaco de Moody con los estudios realizados en modelo y con la aplicación del nuevo criterio.

Con respecto a la Figura 3 se puede comentar lo siguiente: 
1. Con el material del acrílico y lija, los experimentos caen en la zona de transición, así como los resultantes del modelo acrílico-lija con números de Reynolds que varían entre 140000 y 203000.

2. Los modelos correspondientes a plástico y acrílico-plástico caen en la zona de flujo turbulento y algunos resultados en la zona de transición. La variación del número de Reynolds ocurre en un rango de 126000 a 202 000. La aplicación del nuevo criterio con la ecuación de Colebrook es plenamente aplicable a estos modelos.

3. Los modelos con carpeta y acrílico-carpeta caen sensiblemente en una zona de turbulencia desarrollada con números de Reynolds, que varían en un rango de 80000 a 143000 . El criterio aplicable sin ningún problema en estos modelos puede ser el de Nikuradse.

La Figura 4 muestra el Ábaco de Moody ampliado, en el que se pueden apreciar con mayor detalle los resultados obtenidos. 
Tecnología y

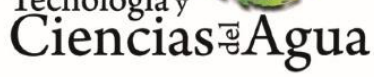

2020, Instituto Mexicano de Tecnología del Agua

Open Access bajo la licencia CC BY-NC-SA 4.0

(https://creativecommons.org/licenses/by-nc-sa/4.0/)

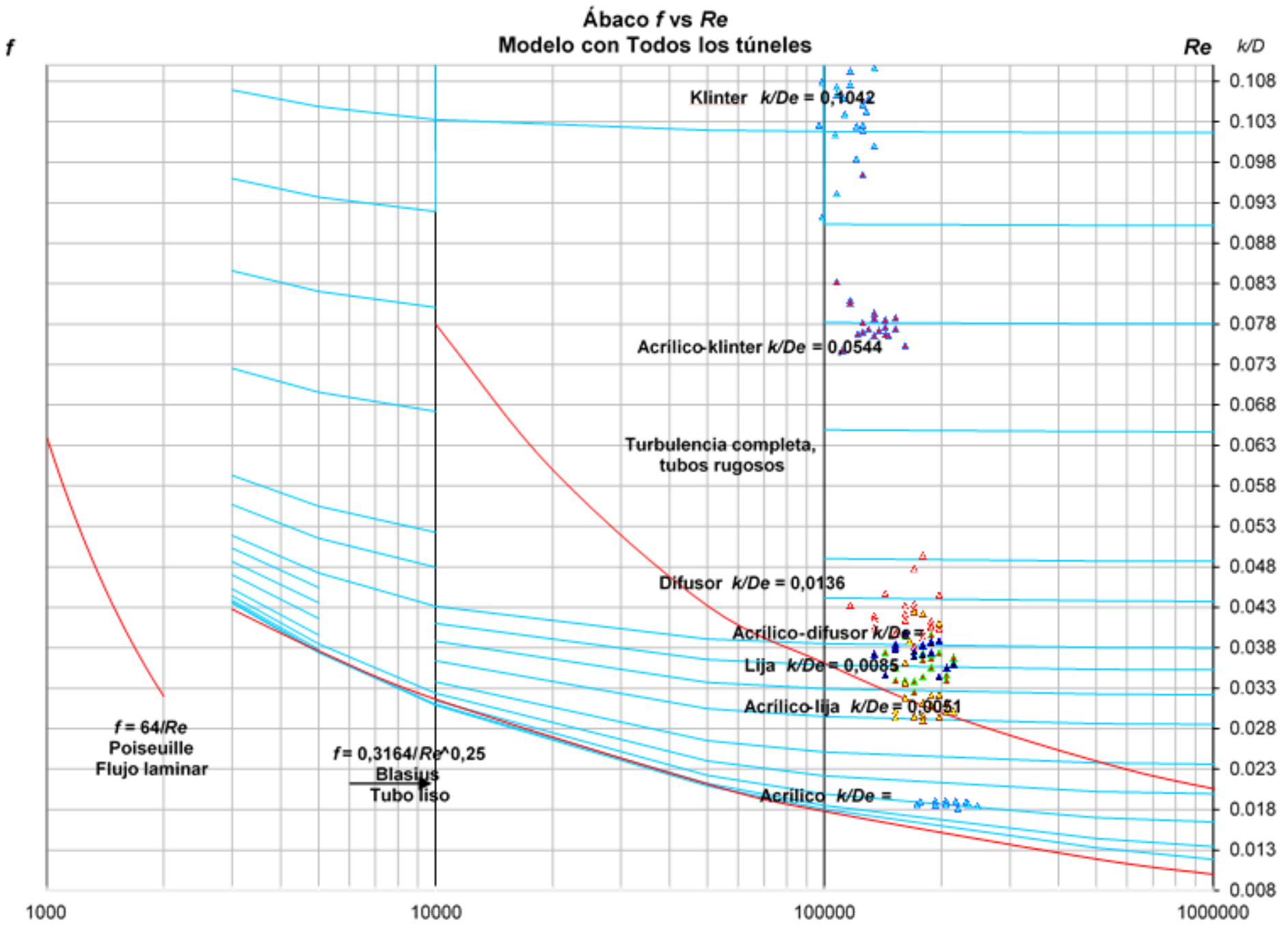

Figura 4. Ábaco de Moody para todos los túneles estudiados.

\section{Análisis de sensibilidad del coeficiente $\lambda$}


Las fuentes de error más comunes en esta experimentación fueron las lecturas de los piezómetros (que influyen en la pérdida de energía), y la medición de la carga en el vertedor triangular aguas abajo en el modelo (que se refleja en el gasto y en consecuencia, en la velocidad media).

Para sensibilizar el análisis de errores, se aplicó la expresión sugerida por Bombardelli (2003):

$\frac{d C_{R}}{C_{R}}=\frac{\partial C_{R}}{\partial h_{f}} d h_{f} \frac{1}{C_{R}}+\frac{\partial C_{R}}{\partial V} d V \frac{1}{C_{R}}$

A la ecuación de Darcy-Weisbach da como resultado:

$$
\frac{d \lambda}{\lambda}=\frac{d h_{f}}{h_{f}}-2 \frac{d V}{V}
$$

En la Tabla 8 y Tabla 9 se muestran los valores obtenidos al aplicar la Ecuación (38) al coeficiente $\lambda$, considerando diferentes variaciones en los errores de la carga hidráulica medida y en la velocidad ( $2 \% \Delta h$ y $3 \% V$; $3 \% \Delta h$ y $3 \% V ; 3 \% \Delta h$ y $4 \% V ; 4 \% \Delta h$ y $4 \% V ; 4 \% \Delta h$ y $5 \% V)$.

Tabla 8. Variación de los coeficientes de fricción al modificar en 2 y $3 \%$ la carga hidráulica y un $3 \%$ la velocidad. 
Tecnología y

Ciencias $₫$ Agua
2020, Instituto Mexicano de Tecnología del Agua

Open Access bajo la licencia CC BY-NC-SA 4.0

(https://creativecommons.org/licenses/by-nc-sa/4.0/)

\begin{tabular}{|c|c|c|c|c|c|c|c|c|c|}
\hline Materiales $(L=3.127 \mathrm{~m})$ & \multicolumn{3}{|c|}{ Experimentales } & \multicolumn{3}{|c|}{ Bombardelli, $2 \% \Delta h$ y $3 \% \Delta V$} & \multicolumn{3}{|c|}{$\begin{array}{c}\text { Bombardelli, } 3 \% \Delta h y \\
3 \% \Delta V\end{array}$} \\
\hline & $\lambda_{\text {mín }}$ & $\lambda_{\text {medio }}$ & $\lambda_{\text {máx }}$ & $\lambda_{\text {mín }}$ & $\lambda$ medio & $\lambda_{\text {máx }}$ & $\lambda_{\text {mín }}$ & $\lambda$ medio & $\lambda_{\text {máx }}$ \\
\hline Acrílico (A) & 0.018 & 0.019 & 0.020 & 0.017 & 0.019 & 0.021 & 0.017 & 0.019 & 0.021 \\
\hline Lija (L) & 0.032 & 0.036 & 0.040 & 0.033 & 0.036 & 0.039 & 0.033 & 0.036 & 0.039 \\
\hline Plástico (P) & 0.036 & 0.042 & 0.049 & 0.039 & 0.042 & 0.045 & 0.038 & 0.042 & 0.046 \\
\hline Carpeta (C) & 0.091 & 0.104 & 0.117 & 0.096 & 0.104 & 0.112 & 0.094 & 0.104 & 0.113 \\
\hline Rugosidad compuesta & $\lambda_{\text {mín }}$ & $\lambda$ medio & $\lambda_{\text {máx }}$ & $\lambda_{\text {mín }}$ & $\lambda$ medio & $\lambda$ máx & $\lambda_{\text {mín }}$ & $\lambda$ medio & $\lambda_{\text {máx }}$ \\
\hline Acrílico-lija (AL) & 0.028 & 0.032 & 0.042 & 0.030 & 0.032 & 0.035 & 0.029 & 0.032 & 0.035 \\
\hline Acrílico-plástico (AP) & 0.034 & 0.038 & 0.042 & 0.035 & 0.038 & 0.041 & 0.034 & 0.038 & 0.041 \\
\hline Acrílico-carpeta (AC) & 0.073 & 0.078 & 0.096 & 0.072 & 0.078 & 0.085 & 0.071 & 0.078 & 0.085 \\
\hline
\end{tabular}

Tabla 9. Variación de los coeficientes de fricción al variar en un 3 y $4 \%$ la carga, y en un 4 y $5 \%$ la velocidad.

\begin{tabular}{|c|c|c|c|c|c|c|c|c|c|c|c|c|}
\hline \multirow{2}{*}{$\begin{array}{c}\text { Materiales ( } L= \\
3.127 \mathrm{~m}) \\
\text { rugosidad } \\
\text { simple }\end{array}$} & \multicolumn{3}{|c|}{ Experimentales } & \multicolumn{3}{|c|}{$\begin{array}{c}\text { Bombardelli, } 3 \% \Delta h y \\
4 \% V\end{array}$} & \multicolumn{3}{|c|}{$\begin{array}{c}\text { Bombardelli, } 4 \% \Delta h y \\
4 \% V\end{array}$} & \multicolumn{3}{|c|}{$\begin{array}{c}\text { Bombardelli, } 4 \% \Delta h y \\
5 \% V\end{array}$} \\
\hline & $\lambda_{\text {mín }}$ & $\lambda$ medio & $\lambda_{\text {máx }}$ & $\lambda_{\text {mín }}$ & $\lambda$ medio & $\lambda_{\text {máx }}$ & $\lambda_{\text {mín }}$ & $\lambda$ medio & $\lambda_{\text {máx }}$ & $\lambda_{\text {mín }}$ & $\lambda$ medio & $\lambda_{\text {máx }}$ \\
\hline Acrílico (A) & 0.018 & 0.019 & 0.020 & 0.017 & 0.019 & 0.021 & 0.017 & 0.019 & 0.021 & 0.016 & 0.019 & 0.022 \\
\hline Lija (L) & 0.032 & 0.036 & 0.040 & 0.032 & 0.036 & 0.040 & 0.031 & 0.036 & 0.040 & 0.031 & 0.036 & 0.041 \\
\hline Plástico (P) & 0.036 & 0.042 & 0.049 & 0.037 & 0.042 & 0.047 & 0.037 & 0.042 & 0.047 & 0.036 & 0.042 & 0.048 \\
\hline Carpeta (C) & 0.091 & 0.104 & 0.117 & 0.092 & 0.104 & 0.115 & 0.091 & 0.104 & 0.116 & 0.089 & 0.104 & 0.116 \\
\hline $\begin{array}{l}\text { Rugosidad } \\
\text { compuesta }\end{array}$ & $\lambda_{\text {mín }}$ & $\lambda_{\text {medio }}$ & $\lambda_{\text {máx }}$ & $\lambda_{\text {mín }}$ & $\lambda_{\text {medio }}$ & $\lambda_{\text {máx }}$ & $\lambda_{\text {mín }}$ & $\lambda$ medio & $\lambda_{\text {máx }}$ & $\lambda_{\text {mín }}$ & $\lambda$ medio & $\lambda_{\text {máx }}$ \\
\hline
\end{tabular}


Tecnología y

Ciencias $\stackrel{\unlhd}{\triangleleft}$ gua
2020, Instituto Mexicano de Tecnología del Agua

Open Access bajo la licencia CCBY-NC-SA 4.0

(https://creativecommons.org/licenses/by-nc-sa/4.0/)

\begin{tabular}{|l|c|c|c|c|c|c|c|c|c|c|c|c|}
\hline Acrílico-lija (AL) & 0.028 & $\mathbf{0 . 0 3 2}$ & 0.042 & 0.029 & $\mathbf{0 . 0 3 2}$ & 0.036 & 0.029 & $\mathbf{0 . 0 3 2}$ & 0.036 & 0.028 & $\mathbf{0 . 0 3 2}$ & 0.037 \\
\hline $\begin{array}{l}\text { Acrílico- } \\
\text { plástico (AP) }\end{array}$ & 0.034 & $\mathbf{0 . 0 3 8}$ & 0.042 & 0.034 & $\mathbf{0 . 0 3 8}$ & 0.042 & 0.033 & $\mathbf{0 . 0 3 8}$ & 0.042 & 0.032 & $\mathbf{0 . 0 3 8}$ & 0.043 \\
\hline $\begin{array}{l}\text { Acrílico-carpeta } \\
\text { (AC) }\end{array}$ & 0.073 & $\mathbf{0 . 0 7 8}$ & 0.096 & 0.070 & $\mathbf{0 . 0 7 8}$ & 0.087 & 0.069 & $\mathbf{0 . 0 7 8}$ & 0.088 & 0.067 & $\mathbf{0 . 0 7 8}$ & 0.089 \\
\hline
\end{tabular}

La variación de la carga hidráulica entre 2 y $4 \%$ permite determinar que ésta no sea significativa en la estimación de los coeficientes de fricción calculados de forma teórica y los medidos de modo experimental.

La variación en la velocidad permite definirque la ecuación de Darcy se vuelva más sensible a la variación de la misma; pero en todos los casos, el cálculo teórico queda muy próximo al experimental, por lo que se valida el planteamiento teórico.

\section{Discusión de resultados}

Los resultados obtenidos permiten destacar lo siguiente: 
1. Se han obtenido con el nuevo modelo de Colebrook presentado en este artículo valores muy cercanos entre lo modelado y lo medido, particularmente en la zona de transición del análisis, ya que el modelo matemático propuesto toma en cuenta la variación de dicha zona, la cual se presenta con frecuencia en el diseño y la construcción de los modelos que se llevan a cabo en la práctica profesional.

2. Por lo general, las variaciones de dicha zona de transición -cuando se construyen modelos- no se consideran, y no se pueden establecer conclusiones importantes de la interpretación entre el modelo y el prototipo.

3. Es conveniente destacar que -en el funcionamiento de prototiposmucho tiempo de su comportamiento sucede en dichas zonas de transición y es de fundamental importancia interpretar los fenómenos que se presentan en los modelos para prever que en el prototipo se presenten zonas de separación o fenómenos transitorios que afectan su comportamiento.

4. Es destacable considerar que desde un punto de vista científico, la aplicación de la ecuación de Colebrook es la más aceptada, y que el planteamiento aquí presentado le permite al diseñador acercarse a una interpretación más realista del comportamiento hidráulico del fenómeno, extrapolando a prototipo lo encontrado. La similitud obtenida entre lo medido y lo obtenido es la mejor dimensión para efectos comparativos del estudio.

5. Debe resaltarse que en la literatura técnica hay muy poco escrito sobre este tema. A partir de las ideas propuestas por el autor 
(Marengo, 2006), en varios túneles de desvío en diversos proyectos del mundo se ha aplicado el concepto de "rugosidad compuesta", aunque en prácticamente todos los casos se analizan los túneles con un comportamiento de turbulencia desarrollada por completo, por lo que se emplean las ecuaciones de Chezy o la de Manning, no la de Colebrook. Los resultados y el criterio en sí mejoran el estado del arte al aplicar conceptos que son poco o nada usados en la práctica profesional por la complejidad inherente del fenómeno estudiado y aportan para alcanzar un mejor conocimiento del mismo.

6. Sería conveniente instrumentar las obras de desvío en prototipo y retroalimentar algunas de las suposiciones hechas durante el desarrollo de este trabajo para conseguir retroalimentación de los estudios realizados.

7. Indudablemente habría que hacer análisis similares con $2 D \circ 3 D$, para validar con lo obtenido el comportamiento real y los modelos físicos.

\section{Conclusiones}

A partir del análisis efectuado se muestran las siguientes conclusiones: 
Al comparar los resultados conseguidos con el nuevo modelo con los calculados de manera experimental se demuestra la validez de la comparación, al calcular los coeficientes de fricción $\lambda_{b}, \lambda_{w}$ y $\lambda_{c}$ al aplicar el Caso I y los calculados con el Caso II.

- A partir de las condiciones de operación hidráulicas de los modelos probados, el coeficiente de fricción $\lambda$, calculado con la ecuación de Colebrook-White adaptada a la geometría de la sección baúl, resulta relevante para el análisis realizado.

- Dentro de los resultados importantes del estudio, los modelos realizados con el material del acrílico y el acrílico-lija caen claramente en la zona de transición del Ábaco de Moody.

- Los túneles probados con el material del plástico y acrílico-plástico caen en la frontera del flujo de transición y la zona de turbulencia de flujo completamente rugoso. El nuevo criterio continúa siendo de gran utilidad para su aplicación en este tipo de experimentos.

- Los túneles que se modelaron con la carpeta y el acrílico-carpeta caen por completo en la zona de turbulencia desarrollada, y entonces es indistinto emplear el nuevo criterio u otro, como el Nikuradse, o bien pueden usarse ecuaciones empíricas para su evaluación.

Dentro del trabajo experimental que debe realizarse más adelante, está el hecho de validar el empleo de los coeficientes de rugosidad estimados con un solo material (lija, plástico o carpeta), y la recomendación de que se obtenga para su uso en modelos de materiales combinados. 
También necesitará validarse el criterio aquí empleado en túneles prototipo, lo que permitirá avanzar en el conocimiento de la mecánica de fluidos en materiales como el concreto hidráulico y el concreto lanzado, así como en la caracterización del flujo en túneles con rugosidad compuesta de gran tamaño.

Como resultado del análisis experimental efectuado, puede decirse que es posible agrupar las rugosidades absolutas por gastos y número de Reynolds, lo cual permite alcanzar mayor semejanza cinemática.

Puede concluirse que el análisis con la ecuación de Colebrook-White es un método más científico que el propuesto por Elfman con la ecuación de Nikuradse; se obtienen resultados más realistas y describe mejor los fenómenos estudiados desde el punto de vista de la mecánica de fluidos.

Sin duda será posible estudiar túneles con rugosidad compuesta aplicables a otras geometrías, como la herradura o circular.

\section{Anexo I}

\section{Tablas del cálculo de validación de los modelos experimentales}


Tecnología y

Ciencias $\approx$ Agua
2020, Instituto Mexicano de Tecnología del Agua

Open Access bajo la licencia CC BY-NC-SA 4.0

(https://creativecommons.org/licenses/by-nc-sa/4.0/)

En la Tabla I.1 se muestran los parámetros hidráulicos para los modelos medidos de acrílico-lija, con pendientes L1-0.001, L2-0.004 y L3-0.008. En cada caso se estimó la rugosidad absoluta evaluada con la ecuación de Colebrook $K_{C}$, que se reporta como valor medido.

Tabla I.1. Resultados de los modelos experimentales acrílico-lija con el valor de la rugosidad absoluta $K_{C}$ medida en modelo.

\begin{tabular}{|c|c|c|c|c|c|c|c|}
\hline$S$ & $Q\left(\mathrm{~m}^{3} / \mathrm{s}\right)$ & $V(\mathrm{~m} / \mathrm{s})$ & $4 R e$ & $I$ & $\tau$ & $\lambda$ medido & $K_{C}$ medido \\
\hline \multirow{5}{*}{$\begin{array}{c}\text { AL1 } \\
0.001\end{array}$} & 0.01700 & 1.08 & 143506 & 0.01206 & 0.40113 & 0.02716 & 0.000404 \\
\hline & 0.01900 & 1.20 & 160389 & 0.01515 & 0.50365 & 0.02730 & 0.000418 \\
\hline & 0.02000 & 1.27 & 168830 & 0.01682 & 0.55943 & 0.02737 & 0.000424 \\
\hline & 0.02200 & 1.39 & 185713 & 0.02023 & 0.67251 & 0.02719 & 0.000417 \\
\hline & 0.02300 & 1.46 & 194155 & 0.02345 & 0.77955 & 0.02884 & 0.000523 \\
\hline \multirow{5}{*}{$\begin{array}{c}\text { AL2 } \\
0.004\end{array}$} & 0.01800 & 1.14 & 151947 & 0.01466 & 0.48731 & 0.02943 & 0.000553 \\
\hline & 0.02000 & 1.27 & 168830 & 0.01783 & 0.59284 & 0.02900 & 0.000528 \\
\hline & 0.02100 & 1.33 & 177272 & 0.01937 & 0.64411 & 0.02858 & 0.000502 \\
\hline & 0.02200 & 1.39 & 185713 & 0.02214 & 0.73607 & 0.02976 & 0.000584 \\
\hline & 0.02400 & 1.52 & 202596 & 0.02468 & 0.82050 & 0.02788 & 0.000462 \\
\hline \multirow{4}{*}{$\begin{array}{c}\text { AL3 } \\
0.008\end{array}$} & 0.01800 & 1.14 & 151947 & 0.01666 & 0.55397 & 0.03346 & 0.000866 \\
\hline & 0.02000 & 1.27 & 168830 & 0.01888 & 0.62785 & 0.03072 & 0.000650 \\
\hline & 0.02100 & 1.33 & 177272 & 0.02178 & 0.72434 & 0.03214 & 0.000773 \\
\hline & 0.02300 & 1.46 & 194155 & 0.02682 & 0.89169 & 0.03299 & 0.000836 \\
\hline
\end{tabular}


Tecnología y

Ciencias $₫$ Agua
2020, Instituto Mexicano de Tecnología del Agua

Open Access bajo la licencia CCBY-NC-SA 4.0

(https://creativecommons.org/licenses/by-nc-sa/4.0/)

\begin{tabular}{|l|l|l|l|l|l|l|l|}
\hline & 0.02400 & 1.52 & 202596 & 0.02863 & 0.95200 & 0.03234 & 0.000784 \\
\hline
\end{tabular}

En la Tabla I.2 se muestran los resultados de la aplicación del nuevo criterio para el Caso I con el material acrílico-lija.

Tabla I.2. Aplicación del Caso I con el nuevo criterio para estimar el $K_{C W}$ calculado en paredes y bóveda del modelo acrílico-lija.

\begin{tabular}{|c|c|c|c|c|c|c|c|c|c|c|}
\hline$S$ & $\mathrm{Q}\left(\mathrm{m}^{3} / \mathrm{s}\right)$ & $\lambda_{\text {medido }}$ & $V(\mathrm{~m} / \mathrm{s})$ & $4 R e$ & $\bar{A}$ & $A_{b}\left(\mathrm{~m}^{2}\right)$ & $A_{w}\left(m^{2}\right)$ & $\lambda b$ & $\lambda w$ & $K_{C W}$ calculado \\
\hline \multirow{5}{*}{$\begin{array}{c}\text { AL1 } \\
0.001\end{array}$} & 0.017 & 0.02720 & 1.077 & 143183 & 0.202 & 0.0032 & 0.0126 & 0.01983 & 0.03007 & 0.000697 \\
\hline & 0.019 & 0.027320 & 1.203 & 160028 & 0.1999 & 0.0031 & 0.0127 & 0.01962 & 0.03029 & 0.000691 \\
\hline & 0.020 & 0.027369 & 1.267 & 168450 & 0.198 & 0.00310 & 0.0127 & 0.0195 & 0.03040 & 0.000706 \\
\hline & 0.022 & 0.027191 & 1.393 & 185295 & 0.197 & 0.00310 & 0.0127 & 0.0193 & 0.03027 & 0.000697 \\
\hline & 0.023 & 0.028838 & 1.457 & 193718 & 0.187 & 0.00290 & 0.0129 & 0.0194 & 0.03251 & 0.000904 \\
\hline \multirow{5}{*}{$\begin{array}{c}\text { AL2 } \\
0.004\end{array}$} & 0.018 & 0.029433 & 1.140 & 151605 & 0.188 & 0.0030 & 0.0128 & 0.0202 & 0.03309 & 0.000948 \\
\hline & 0.020 & 0.029004 & 1.267 & 168450 & 0.188 & 0.0030 & 0.0128 & 0.0197 & 0.03261 & 0.000907 \\
\hline & 0.021 & 0.028582 & 1.330 & 176873 & 0.190 & 0.00280 & 0.0130 & 0.01956 & 0.03209 & 0.000859 \\
\hline & 0.022 & 0.029761 & 1.393 & 185295 & 0.183 & 0.00290 & 0.01290 & 0.01961 & 0.033371 & 0.001020 \\
\hline & 0.024 & 0.027876 & 1.520 & 202140 & 0.191 & 0.0030 & 0.0128 & 0.0192 & 0.031240 & 0.000788 \\
\hline \multirow{5}{*}{$\begin{array}{c}\text { AL3 } \\
0.008\end{array}$} & 0.018 & 0.032002 & 1.140 & 151605 & 0.176 & 0.00280 & 0.0130 & 0.02032 & 0.03654 & 0.001326 \\
\hline & 0.0200 & 0.03072 & 1.267 & 168450 & 0.180 & 0.00280 & 0.0130 & 0.01993 & 0.034920 & 0.001460 \\
\hline & 0.0210 & 0.032143 & 1.330 & 176873 & 0.172 & 0.00270 & 0.0131 & 0.02002 & 0.03686 & 0.001375 \\
\hline & 0.0230 & 0.032987 & 1.457 & 193718 & 0.167 & 0.00260 & 0.0132 & 0.01992 & 0.03807 & 0.001530 \\
\hline & 0.0240 & 0.032344 & 1.520 & 202140 & 0.169 & 0.00270 & 0.01310 & 0.01976 & 0.037240 & 0.001429 \\
\hline
\end{tabular}


Tecnología y

Ciencias $\approx$ Agua
2020, Instituto Mexicano de Tecnología del Agua

Open Access bajo la licencia CC BY-NC-SA 4.0

(https://creativecommons.org/licenses/by-nc-sa/4.0/)

En la Tabla I.3 se muestran los resultados de la aplicación del Caso II para el modelo acrílico-lija, con las pendientes AL1-0.001, AL2-0.004 y AL3-0.008; se encuentran los valores de la rugosidad absoluta $K_{C}$ validado.

Tabla I.3. Valores del $K_{C}$ validado para el modelo acrílico-lija.

\begin{tabular}{|c|c|c|c|c|c|c|c|c|c|c|c|}
\hline$S$ & $\begin{array}{c}Q \\
\left(\mathrm{~m}^{3} / \mathrm{s}\right)\end{array}$ & $\begin{array}{c}K c w \\
\text { calculado }\end{array}$ & $\begin{array}{c}V \\
(\mathrm{~m} / \mathrm{s})\end{array}$ & $4 R e$ & $\alpha$ & $\begin{array}{c}A_{b} \\
\left(\mathrm{~m}^{2}\right)\end{array}$ & $\begin{array}{c}A_{w} \\
\left(\mathrm{~m}^{2}\right)\end{array}$ & $\lambda b$ & $\lambda w$ & $\lambda$ & $\begin{array}{l}K_{C} \text { medio } \\
\text { (validado) }\end{array}$ \\
\hline \multirow{5}{*}{$\begin{array}{c}\text { AL1 } \\
0.001\end{array}$} & 0.017 & 0.000697 & 1.077 & 143183 & 0.202 & 0.0032 & 0.0126 & 0.0198 & 0.03044 & 0.02720 & 0.000423 \\
\hline & 0.019 & 0.000691 & 1.203 & 160028 & 0.1999 & 0.0031 & 0.0127 & 0.0196 & 0.03029 & 0.02732 & 0.000417 \\
\hline & 0.020 & 0.000706 & 1.267 & 168450 & 0.198 & 0.0031 & 0.0127 & 0.0195 & 0.03043 & 0.02737 & 0.000424 \\
\hline & 0.022 & 0.000697 & 1.393 & 185295 & 0.197 & 0.0031 & 0.0127 & 0.0193 & 0.03027 & 0.02712 & 0.000417 \\
\hline & 0.023 & 0.000904 & 1.457 & 193718 & 0.187 & 0.0029 & 0.0129 & 0.0194 & 0.03251 & 0.02884 & 0.000523 \\
\hline \multirow{5}{*}{$\begin{array}{c}\text { AL2 } \\
0.004\end{array}$} & 0.018 & 0.000948 & 1.140 & 151605 & 0.188 & 0.0030 & 0.0128 & 0.0202 & 0.03309 & 0.02943 & 0.000553 \\
\hline & 0.020 & 0.000907 & 1.267 & 168450 & 0.188 & 0.0030 & 0.0128 & 0.0197 & 0.03261 & 0.02906 & 0.000528 \\
\hline & 0.021 & 0.000859 & 1.330 & 176873 & 0.190 & 0.0030 & 0.0128 & 0.0196 & 0.03209 & 0.02858 & 0.000502 \\
\hline & 0.022 & 0.001020 & 1.393 & 185295 & 0.183 & 0.0030 & 0.0128 & 0.0196 & 0.03369 & 0.02974 & 0.000583 \\
\hline & 0.024 & 0.000788 & 1.520 & 202140 & 0.191 & 0.0030 & 0.0128 & 0.0192 & 0.03124 & 0.02787 & 0.000462 \\
\hline \multirow{5}{*}{$\begin{array}{c}\text { AL3 } \\
0.008\end{array}$} & 0.018 & 0.001326 & 1.140 & 151605 & 0.176 & 0.0028 & 0.0130 & 0.0203 & 0.03654 & 0.03202 & 0.000745 \\
\hline & 0.020 & 0.001460 & 1.267 & 168450 & 0.171 & 0.0027 & 0.0131 & 0.0202 & 0.03752 & 0.03271 & 0.000807 \\
\hline & 0.0210 & 0.001375 & 1.330 & 176873 & 0.172 & 0.0027 & 0.0131 & 0.0200 & 0.03686 & 0.03214 & 0.000773 \\
\hline & 0.0230 & 0.001530 & 1.457 & 193718 & 0.167 & 0.0026 & 0.0132 & 0.0199 & 0.03807 & 0.03298 & 0.000835 \\
\hline & 0.0240 & 0.001429 & 1.520 & 202140 & 0.169 & 0.0027 & 0.0131 & 0.0199 & 0.03724 & 0.03234 & 0.000784 \\
\hline
\end{tabular}


Tecnología y

Ciencias $₫$ Agua
2020, Instituto Mexicano de Tecnología del Agua

Open Access bajo la licencia CC BY-NC-SA 4.0

(https://creativecommons.org/licenses/by-nc-sa/4.0/)

De la misma manera, en la Tabla I.4 se muestran los valores del modelo acrílico-plástico, en el que en cada caso se estimó la rugosidad absoluta evaluada con la ecuación de Colebrook $K_{c}$ medida, que se reporta como valor medido.

Tabla I.4. Resultados de los modelos experimentales acrílico-plástico con el valor de la rugosidad absoluta $K_{c}$ medida en modelo.

\begin{tabular}{|c|c|c|c|c|c|c|c|}
\hline$S$ & $Q\left(\mathrm{~m}^{3} / \mathrm{s}\right)$ & $V(\mathrm{~m} / \mathrm{s})$ & $4 R e$ & $I$ & $\tau$ & $\lambda$ medido & $K_{C}$ medide \\
\hline \multirow{3}{*}{ AP1 } & 0.0160 & 1.01 & 134760 & 0.01299 & 0.43186 & 0.033013 & 0.000822 \\
\hline & 0.0170 & 1.08 & 143183 & 0.01565 & 0.52023 & 0.035227 & 0.001023 \\
\hline & 0.0190 & 1.20 & 160028 & 0.01891 & 0.62879 & 0.034086 & 0.000923 \\
\hline \multirow{2}{*}{0.001} & 0.0200 & 1.27 & 168450 & 0.02177 & 0.72377 & 0.035409 & 0.001047 \\
\hline & 0.0210 & 1.33 & 176873 & 0.02431 & 0.80820 & 0.035864 & 0.001092 \\
\hline \multirow{3}{*}{ AP2 } & 0.0170 & 1.08 & 143183 & 0.01579 & 0.52500 & 0.035550 & 0.001053 \\
\hline & 0.0180 & 1.14 & 151605 & 0.01837 & 0.61094 & 0.036900 & 0.001188 \\
\hline & 0.0200 & 1.27 & 168450 & 0.02109 & 0.70140 & 0.034315 & 0.000946 \\
\hline \multirow{2}{*}{0.004} & 0.0210 & 1.33 & 176873 & 0.02345 & 0.77980 & 0.034604 & 0.000974 \\
\hline & 0.0230 & 1.46 & 193718 & 0.03121 & 1.03761 & 0.038385 & 0.001350 \\
\hline \multirow{3}{*}{ AP3 } & 0.0180 & 1.14 & 151605 & 0.01743 & 0.57964 & 0.035010 & 0.001005 \\
\hline & 0.0200 & 1.27 & 168450 & 0.02111 & 0.70176 & 0.034333 & 0.000948 \\
\hline & 0.0210 & 1.33 & 176873 & 0.02419 & 0.80429 & 0.035690 & 0.001075 \\
\hline \multirow{2}{*}{0.008} & 0.0220 & 1.39 & 185295 & 0.02673 & 0.88872 & 0.035933 & 0.001100 \\
\hline & 0.0240 & 1.52 & 202140 & 0.03174 & 1.05547 & 0.035859 & 0.001096 \\
\hline
\end{tabular}


Tecnología y

Ciencias $\approx$ Agua
2020, Instituto Mexicano de Tecnología del Agua

Open Access bajo la licencia CC BY-NC-SA 4.0

(https://creativecommons.org/licenses/by-nc-sa/4.0/)

En la Tabla I.5 se muestran los resultados de la aplicación del nuevo criterio para el Caso I con el material acrílico-plástico, donde se reporta el valor de $\mathbf{K} \mathbf{c w}$ calculado en muros y bóveda.

Tabla I.5. Aplicación del Caso I con el nuevo criterio para estimar el

$K_{C w}$ calculado en paredes y bóveda del modelo acrílico-plástico.

\begin{tabular}{|c|c|c|c|c|c|c|c|c|c|c|}
\hline$S$ & $Q\left(\mathrm{~m}^{3} / \mathrm{s}\right)$ & $\bar{\lambda}$ & $V(\mathrm{~m} / \mathrm{s})$ & $4 R e$ & $\bar{A}$ & $\overline{A_{b}\left(\mathrm{~m}^{2}\right)}$ & $\overline{A_{w}\left(\mathrm{~m}^{2}\right)}$ & $\lambda b$ & $\lambda w$ & $K c w$ calculado \\
\hline \multirow{3}{*}{ AP1 } & 0.016 & 0.033013 & 1.013 & 134760 & 0.173 & 0.0027 & 0.0131 & 0.02074 & 0.0382 & 0.001520 \\
\hline & 0.017 & 0.035227 & 1.076 & 143183 & 0.164 & 0.0026 & 0.0132 & 0.02082 & 0.04083 & 0.001880 \\
\hline & 0.019 & 0.034086 & 1.203 & 160028 & 0.166 & 0.0026 & 0.0132 & 0.02045 & 0.03940 & 0.001690 \\
\hline \multirow{2}{*}{0.001} & 0.020 & 0.035409 & 1.266 & 168450 & 0.160 & 0.0025 & 0.0133 & 0.02049 & 0.04120 & 0.001947 \\
\hline & 0.021 & 0.035864 & 1.329 & 176873 & 0.158 & 0.0025 & 0.0133 & 0.02041 & 0.04158 & 0.002045 \\
\hline \multirow{4}{*}{$\begin{array}{c}\text { AP2 } \\
0.004\end{array}$} & 0.017 & 0.035550 & 1.076 & 143183 & 0.163 & 0.0026 & 0.0132 & 0.02085 & 0.04127 & 0.001940 \\
\hline & 0.020 & 0.034315 & 1.266 & 168450 & 0.164 & 0.0026 & 0.0132 & 0.02037 & 0.03974 & 0.001741 \\
\hline & 0.021 & 0.034604 & 1.329 & 176873 & 0.162 & 0.0025 & 0.0133 & 0.02029 & 0.04045 & 0.001841 \\
\hline & 0.023 & 0.038385 & 1.456 & 193718 & 0.148 & 0.0023 & 0.0135 & 0.02048 & 0.04535 & 0.002591 \\
\hline & 0.018 & 0.035010 & 1.139 & 151605 & 0.164 & 0.0026 & 0.0135 & 0.02065 & 0.04060 & 0.001850 \\
\hline & 0.020 & 0.034333 & 1.267 & 168450 & 0.164 & 0.0026 & 0.0135 & 0.02037 & 0.03976 & 0.001744 \\
\hline & 0.021 & 0.035690 & 1.330 & 176873 & 0.158 & 0.0025 & 0.0133 & 0.02042 & 0.04163 & 0.002010 \\
\hline & 0.022 & 0.035933 & 1.457 & 185295 & 0.157 & 0.0025 & 0.0133 & 0.02032 & 0.04201 & 0.002067 \\
\hline & 0.024 & 0.035859 & 1.520 & 202140 & 0.156 & 0.0024 & 0.0134 & 0.02013 & 0.04198 & 0.002070 \\
\hline
\end{tabular}

Para la pendiente AP-001, a partir del gasto $Q=0.020 \mathrm{~m}^{3} / \mathrm{s}, V=$ $1.266 \mathrm{~m} / \mathrm{s} ; R e=168450 ; \lambda=0.035409$. Considerando en la plantilla una rugosidad con valor $K_{b}=0.0000387 \mathrm{~mm}$ se obtiene en el análisis un valor 
$\alpha=0.160$, para el cual, $A_{b}=0.0025, A_{w}=0.0133, \lambda_{b}=0.02049, \lambda_{w}=0.04126$, y el resultado de la rugosidad para paredes y bóveda es $K_{w}=0.001947$.

Debe señalarse que, en los modelos estudiados con el material sólo del acrílico, el coeficiente de rugosidad es de $\lambda=0.0177$; el incremento a un valor $\lambda_{b}=0.02049$, puede explicarse por la influencia de la rugosidad en las paredes y bóveda.

En la Tabla I. 6 se muestran los resultados de la aplicación del Caso II para el modelo acrílico-plástico con las pendientes AP1-0.001, AP20.004 , y AP3-0.008; se encuentran los valores del $K_{C}$ validado.

Tabla I.6. Validación del criterio para el Caso II del modelo acrílicoplástico. 
Tecnología y

Ciencias $\stackrel{\unlhd}{\unlhd}$ gua
2020, Instituto Mexicano de Tecnología del Agua

Open Access bajo la licencia CC BY-NC-SA 4.0

(https://creativecommons.org/licenses/by-nc-sa/4.0/)

\begin{tabular}{|c|c|c|c|c|c|c|c|c|c|c|c|}
\hline$S$ & $Q\left(m^{3} / s\right)$ & $\begin{array}{c}\text { KCW } \\
\text { calculado }\end{array}$ & $V(m / s)$ & $4 R e$ & $\alpha$ & $A_{b}\left(m^{2}\right)$ & $A_{w}\left(m^{2}\right)$ & $\lambda b$ & $\lambda w$ & $\lambda$ & $K_{c}$ medio (validado) \\
\hline \multirow{5}{*}{$\begin{array}{l}\text { AP1 } \\
0.001\end{array}$} & 0.016 & 0.001520 & 1.013 & 134760 & 0.173 & 0.0027 & 0.0131 & 0.020744 & 0.038197 & 0.033309 & 0.000847 \\
\hline & 0.017 & 0.001880 & 1.076 & 143183 & 0.164 & 0.0026 & 0.0132 & 0.020823 & 0.040839 & 0.035233 & 0.001023 \\
\hline & 0.019 & 0.001690 & 1.203 & 160028 & 0.166 & 0.0026 & 0.0132 & 0.020451 & 0.039390 & 0.034086 & 0.000923 \\
\hline & 0.020 & 0.001947 & 1.266 & 168450 & 0.160 & 0.0025 & 0.0133 & 0.024850 & 0.041217 & 0.035411 & 0.001047 \\
\hline & 0.021 & 0.002045 & 1.329 & 176873 & 0.158 & 0.0025 & 0.0133 & 0.020408 & 0.04187 & 0.035865 & 0.001092 \\
\hline \multirow{5}{*}{$\begin{array}{c}\text { AP2 } \\
0.004\end{array}$} & 0.017 & 0.001940 & 1.076 & 143183 & 0.163 & 0.0026 & 0.0132 & 0.020851 & 0.041264 & 0.035547 & 0.001053 \\
\hline & 0.018 & 0.002220 & 1.139 & 151605 & 0.157 & 0.0025 & 0.0133 & 0.020867 & 0.043137 & 0.03691 & 0.00187 \\
\hline & 0.020 & 0.001741 & 1.266 & 168450 & 0.164 & 0.0026 & 0.0132 & 0.020370 & 0.039738 & 0.03431 & 0.000946 \\
\hline & 0.021 & 0.001841 & 1.329 & 176873 & 0.162 & 0.0025 & 0.0132 & 0.020289 & 0.04053 & 0.034806 & 0.000992 \\
\hline & 0.023 & 0.002591 & 1.456 & 193718 & 0.148 & 0.0023 & 0.0135 & 0.020483 & 0.045337 & 0.038387 & 0.00135 \\
\hline \multirow{5}{*}{$\begin{array}{l}\text { AP3 } \\
0.008\end{array}$} & 0.018 & 0.001850 & 1.139 & 151605 & 0.164 & 0.0026 & 0.0132 & 0.020654 & 0.040598 & 0.035013 & 0.001005 \\
\hline & 0.020 & 0.001744 & 1.267 & 168450 & 0.164 & 0.0026 & 0.0132 & 0.020369 & 0.039761 & 0.034331 & 0.000947 \\
\hline & 0.021 & 0.002010 & 1.330 & 176873 & 0.158 & 0.0025 & 0.0133 & 0.020416 & 0.041626 & 0.035656 & 0.001075 \\
\hline & 0.022 & 0.002067 & 1.457 & 185295 & 0.157 & 0.0025 & 0.0133 & 0.020316 & 0.042005 & 0.035931 & 0.00110 \\
\hline & 0.024 & 0.002070 & 1.520 & 202140 & 0.156 & 0.0024 & 0.0133 & 0.020125 & 0.041989 & 0.035866 & 0.001097 \\
\hline
\end{tabular}

Análogamente, en la Tabla I.7 se muestran los resultados medidos en el modelo acrílico-carpeta con las pendientes AC1-0.001, AC2-0.004 y AC3-0.008.

Tabla I.7. Resultados de los valores medidos en forma experimental para el modelo acrílico-carpeta. 
Teçnología y

Ciencias $\approx$ Agua
2020, Instituto Mexicano de Tecnología del Agua

Open Access bajo la licencia CC BY-NC-SA 4.0

(https://creativecommons.org/licenses/by-nc-sa/4.0/)

\begin{tabular}{|c|c|c|c|c|c|c|c|}
\hline$s$ & $Q\left(\mathrm{~m}^{3} / \mathrm{s}\right)$ & $V(\mathrm{~m} / \mathrm{s})$ & $4 R e$ & $I$ & $\tau$ & $\lambda$ medido & $K_{C \text { medido }}$ \\
\hline \multirow{5}{*}{ AC1 0.001} & 0.0124 & 0.785 & 104439 & 0.01633 & 0.54285 & 0.06909 & 0.00613 \\
\hline & 0.0136 & 0.861 & 114546 & 0.02018 & 0.67100 & 0.07099 & 0.00651 \\
\hline & 0.0145 & 0.918 & 122127 & 0.02313 & 0.76900 & 0.07158 & 0.00663 \\
\hline & 0.0154 & 0.975 & 129707 & 0.02603 & 0.86549 & 0.07142 & 0.00660 \\
\hline & 0.0163 & 1.032 & 137287 & 0.02893 & 0.96198 & 0.07086 & 0.00649 \\
\hline \multirow{5}{*}{ AC2 0,004} & 0.0110 & 0.697 & 92648 & 0.01293 & 0.43001 & 0.06955 & 0.00621 \\
\hline & 0.0120 & 0.760 & 101070 & 0.01602 & 0.53254 & 0.07237 & 0.00678 \\
\hline & 0.0130 & 0.823 & 109493 & 0.01937 & 0.64411 & 0.07458 & 0.00724 \\
\hline & 0.0140 & 0.887 & 117915 & 0.02146 & 0.71346 & 0.07123 & 0.00656 \\
\hline & 0.0150 & 0.950 & 126338 & 0.02450 & 0.81447 & 0.07084 & 0.00648 \\
\hline \multirow{5}{*}{ AC3 0,008} & 0.0130 & 0.823 & 109493 & 0.01870 & 0.62181 & 0.07200 & 0.00671 \\
\hline & 0.0140 & 0.887 & 117915 & 0.02178 & 0.72434 & 0.07232 & 0.00678 \\
\hline & 0.0150 & 0.950 & 126338 & 0.02519 & 0.83741 & 0.07283 & 0.00689 \\
\hline & 0.0160 & 1.013 & 134760 & 0.02859 & 0.95049 & 0.07266 & 0.00685 \\
\hline & 0.0170 & 1.077 & 143183 & 0.03181 & 1.05754 & 0.07161 & 0.006643 \\
\hline
\end{tabular}

En la Tabla I. 8 se muestran los resultados de la aplicación del nuevo criterio para el Caso I con el material acrílico-carpeta, donde se reporta el valor de $K_{C} w$ calculado en muros y bóveda.

Tabla I.8. Aplicación del Caso I, con el nuevo criterio para el acrílicocarpeta. 
Tecnología y

Ciencias $₫$ Agua
2020, Instituto Mexicano de Tecnología del Agua

Open Access bajo la licencia CC BY-NC-SA 4.0

(https://creativecommons.org/licenses/by-nc-sa/4.0/)

\begin{tabular}{|c|c|c|c|c|c|c|c|c|c|c|}
\hline$S$ & $Q\left(\mathrm{~m}^{3} / \mathrm{s}\right)$ & $\lambda$ & $\begin{array}{c}V \\
(\mathrm{~m} / \mathrm{s})\end{array}$ & $4 R e$ & $\alpha$ & $\begin{array}{c}A_{b} \\
\left(\mathrm{~m}^{2}\right)\end{array}$ & $A_{w}\left(m^{2}\right)$ & $\lambda b$ & $\lambda w$ & $K_{C W}$ calculado \\
\hline \multirow{5}{*}{$\begin{array}{l}\text { AC1 } \\
0.001\end{array}$} & 0.0124 & 0.06909 & 0.785 & 104439 & 0.099 & 0.0015 & 0.0142 & 0.024693 & 0.08636 & 0.012240 \\
\hline & 0.0136 & 0.07099 & 0.861 & 114546 & 0.096 & 0.0015 & 0.0143 & 0.024503 & 0.08967 & 0.013049 \\
\hline & 0.0145 & 0.07158 & 0.918 & 122127 & 0.095 & 0.0015 & 0.0143 & 0.024310 & 0.08997 & 0.013320 \\
\hline & 0.0154 & 0.07142 & 0.975 & 129707 & 0.094 & 0.0015 & 0.0143 & 0.024160 & 0.08980 & 0.01329 \\
\hline & 0.0163 & 0.07086 & 1.032 & 137287 & 0.094 & 0.0015 & 0.0143 & 0.023950 & 0.08911 & 0.013093 \\
\hline \multirow{5}{*}{$\begin{array}{l}\text { AC2 } \\
0.004\end{array}$} & 0.0110 & 0.06955 & 0.697 & 92648 & 0.100 & 0.0016 & 0.0142 & 0.024731 & 0.08683 & 0.012351 \\
\hline & 0.0120 & 0.07237 & 0.760 & 101070 & 0.096 & 0.0015 & 0.0143 & 0.025020 & 0.090792 & 0.013533 \\
\hline & 0.0130 & 0.07458 & 0.823 & 109493 & 0.093 & 0.0015 & 0.0143 & 0.020488 & 0.093910 & 0.01448 \\
\hline & 0.0140 & 0.07123 & 0.887 & 117915 & 0.095 & 0.0015 & 0.0143 & 0.024473 & 0.089420 & 0.01316 \\
\hline & 0.0150 & 0.07084 & 0.950 & 126338 & 0.095 & 0.0015 & 0.0143 & 0.024191 & 0.088979 & 0.013042 \\
\hline \multirow{5}{*}{$\begin{array}{c}\text { AC3 } \\
0.008\end{array}$} & 0.0130 & 0.07200 & 0.823 & 109493 & 0.096 & 0.0015 & 0.0143 & 0.02467 & 0.090411 & 0.013428 \\
\hline & 0.0140 & 0.07232 & 0.887 & 117915 & 0.094 & 0.0015 & 0.0143 & 0.024531 & 0.09091 & 0.013603 \\
\hline & 0.0150 & 0.07283 & 0.950 & 126338 & 0.093 & 0.0015 & 0.0143 & 0.024325 & 0.01969 & 0.013848 \\
\hline & 0.0160 & 0.07266 & 1.013 & 104439 & 0.092 & 0.0014 & 0.0144 & 0.02416 & 0.09153 & 0.013815 \\
\hline & 0.0170 & 0.07161 & 1.077 & 143783 & 0.093 & 0.0015 & 0.0143 & 0.02386 & 0.09019 & 0.013417 \\
\hline
\end{tabular}

En la Tabla I.9 se muestran los resultados de la aplicación del Caso II para el modelo acrílico-carpeta con las pendientes AC1-0.001, AC20.004 y AC3-0.008 donde se encuentran los valores del $K_{C}$ validado. 
Tecnología y

Ciencias $₫$ Agua
2020, Instituto Mexicano de Tecnología del Agua

Open Access bajo la licencia CC BY-NC-SA 4.0

(https://creativecommons.org/licenses/by-nc-sa/4.0/)

Tabla I.9. Validación del criterio para el Caso II del modelo acrílicocarpeta.

\begin{tabular}{|c|c|c|c|c|c|c|c|c|c|c|c|}
\hline$S$ & $Q\left(m^{3} / s\right)$ & $\begin{array}{c}\text { KCw } \\
\text { calculado }\end{array}$ & $V(\mathrm{~m} / \mathrm{s})$ & $4 R e$ & $\alpha$ & $A_{b}\left(m^{2}\right)$ & $A_{w}\left(m^{2}\right)$ & $\lambda b$ & $\lambda w$ & $\lambda$ & $K_{c}$ medio (validado) \\
\hline \multirow{5}{*}{ AC1 0.001} & 0.0124 & 0.012240 & 0.785 & 104439 & 0.099 & 0.0015 & 0.0142 & 0.024693 & 0.08636 & 0.06909 & 0.00613 \\
\hline & 0.0136 & 0.013049 & 0.861 & 114546 & 0.096 & 0.0015 & 0.0143 & 0.024503 & 0.08967 & 0.07099 & 0.00651 \\
\hline & 0.0145 & 0.013320 & 0.918 & 122127 & 0.095 & 0.0015 & 0.0143 & 0.024310 & 0.08997 & 0.07158 & 0.00663 \\
\hline & 0.0154 & 0.01329 & 0.975 & 129707 & 0.094 & 0.0015 & 0.0143 & 0.024160 & 0.08980 & 0.07142 & 0.00660 \\
\hline & 0.0163 & 0.013093 & 1.032 & 137287 & 0.094 & 0.0015 & 0.0143 & 0.023950 & 0.08911 & 0.07086 & 0.00649 \\
\hline \multirow{5}{*}{ AC2 0.004} & 0.0110 & 0.012351 & 0.697 & 92648 & 0.100 & 0.0016 & 0.0142 & 0.024731 & 0.08683 & 0.06955 & 0.00621 \\
\hline & 0.0120 & 0.013533 & 0.760 & 101070 & 0.096 & 0.0015 & 0.0143 & 0.025020 & 0.090792 & 0.07237 & 0.00678 \\
\hline & 0.0130 & 0.01448 & 0.823 & 109493 & 0.093 & 0.0015 & 0.0143 & 0.020488 & 0.093910 & 0.07458 & 0.00724 \\
\hline & 0.0140 & 0.01316 & 0.887 & 117915 & 0.095 & 0.0015 & 0.0143 & 0.024473 & 0.089420 & 0.07123 & 0.00656 \\
\hline & 0.0150 & 0.013042 & 0.950 & 126338 & 0.095 & 0.0015 & 0.0143 & 0.024191 & 0.088979 & 0.07084 & 0.00648 \\
\hline \multirow{5}{*}{ AC3 0.008} & 0.0130 & 0.013428 & 0.823 & 109493 & 0.096 & 0.0015 & 0.0143 & 0.02467 & 0.090411 & 0.07200 & 0.00671 \\
\hline & 0.0140 & 0.013603 & 0.887 & 117915 & 0.094 & 0.0015 & 0.0143 & 0.024531 & 0.09091 & 0.07232 & 0.00678 \\
\hline & 0.0150 & 0.013848 & 0.950 & 126338 & 0.093 & 0.0015 & 0.0143 & 0.024325 & 0.01969 & 0.07283 & 0.00689 \\
\hline & 0.0160 & 0.013815 & 1.013 & 104439 & 0.092 & 0.0014 & 0.0144 & 0.02416 & 0.09153 & 0.07266 & 0.00685 \\
\hline & 0.0170 & 0.013417 & 1.077 & 143783 & 0.093 & 0.0015 & 0.0143 & 0.02386 & 0.09019 & 0.07161 & 0.006643 \\
\hline
\end{tabular}

Nota: los autores agradecen las amables sugerencias de los revisores anónimos.

\section{Notación empleada:}

$V=$ velocidad media .

$V_{b}$ y $V_{w}=$ velocidad media en piso, paredes y bóveda. 
Tecnología y

Ciencias $₫$ Agua
2020, Instituto Mexicano de Tecnología del Agua

Open Access bajo la licencia CC BY-NC-SA 4.0

(https://creativecommons.org/licenses/by-nc-sa/4.0/)

$R=$ radio hidráulico

$R e=$ número de Reynolds.

$\tau=$ esfuerzo cortante que ocasiona el flujo.

$g=$ aceleración de la gravedad .

$S=$ gradiente hidráulico .

$P_{b}$ y $P_{w}=$ perímetro mojado del piso, paredes y bóveda.

$P=$ perímetro mojado.

$A_{b}$ y $A_{w}=$ área hidráulica del piso, paredes y bóveda.

$A$ = área de la sección transversal.

$Q=$ gasto analizado .

$Q_{b}$ y $Q_{w}=$ gasto analizado en el piso, paredes y bóveda.

$\rho=$ densidad del flujo.

$F=$ fuerza cortante total.

$\tau_{b}$ y $\tau_{w}=$ esfuerzos cortantes en piso, paredes y bóveda.

$\Delta H_{b}$ y $\Delta H_{w}=$ cargas piezométricas en piso, paredes y bóveda.

$K_{b}$ y $K_{w}=$ coeficientes de rugosidad en piso, paredes y bóveda.

$v=$ viscosidad cinemática del flujo.

\section{Referencias}


Tecnología y

Ciencias Agua
2020, Instituto Mexicano de Tecnología del Agua

Open Access bajo la licencia CC BY-NC-SA 4.0

(https://creativecommons.org/licenses/by-nc-sa/4.0/)

Bombardelli, F. A. (2003). Hydraulic design of large diameter pipes. Journal of Hydraulic Engineering, 129(11), 785-793.

Chow, V. T. (1959). Open channel hydraulics. New York, USA: McGrawHill.

Churchill, S. (1973). Empirical expressions for the shear in turbulent flow in commercial pipes. Journal of American Institute of Chemical, $19(2), 375-376$.

Colebrook, C. F. (1939). Turbulent flow in pipes with particular reference to the transition region between smooth and rough pipe laws. Journal of the Institution of Civil Engineers, 11(4), 133-156.

Czarnota, Z. (1986). Hydraulics of rock tunnels. Stockholm, Sweden: The Royal Institute of Technology.

Elfman, S. (2005). Hydropower tunnels: Estimation of head losses. Dam Engineering, 5(4), 256-264. Great Britain.

Haaland, S.E. «Simple and explicit formulas for the friction factor in turbulent pipe flow.» Journal of Fluids Engineering 105, no 1 (1983): 89-90.

Leopardi, M. (2005). On roughness similarity of hydraulic models. Journal of Hydraulic Research, 42(33), 239-245.

Marengo-Mogollón, H. (2006). Case study: Dam safety during construction, lessons of the overtopping diversion works at Aguamilpa Dam. Journal of Hydraulic Engineering, 132(11), 11211127. DOI: $10.1061 /($ ASCE)0733-9429(2006)132:11(1121)

Marengo-Mogollón, H. (2016). Composite roughness in hydraulic models. Tecnología y ciencias del agua, 7(6), 159-178. 
Nikuradse, J. (1933). Strömungsgesetze in rauhen Rohren. Berlín, Deutchland: VDI-Verlag.

Nikuradse, J. (1933) Strömungsgesetze in rauhenRohren, VDI-Verlag, Berlín.

Swamee, P. A. (1976). Explicit equations for pipe-flow problems. Journal of Hydraulics Division, 102(5), 657-664.

USBR-US Bureau of Reclamation, 1992. Friction factors forlarge conduits flowing full. Boulder City (NV): USBR.

Yen, B. C. «Dimensionally homogeneous Mannings' formula.» Journal of Hydraulic Engineering 1, no 128 (1992): 1326-1332.

Yen, C. B. (2002). Open channel flow resistance. Journal of Hydraulic Engineering, 128(20), 20-39. DOI: 10.1061/AASCE)073339429(2002)128:1(20) 